\title{
Explaining two circumnuclear star forming rings in NGC 5248 ${ }^{\star}$
}

\author{
T. P. R. van der Laan ${ }^{1,2}$, E. Schinnerer ${ }^{1}$, E. Emsellem ${ }^{3}$, S. Meidt ${ }^{1}$, G. Dumas ${ }^{2}$, T. Böker ${ }^{4}$, L. Hunt ${ }^{5}$, S. Haan ${ }^{6}$, \\ C. Mundell ${ }^{7}$, and H. Wozniak ${ }^{8}$
}

\author{
1 Max-Planck-Institut für Astronomie, Königstuhl 17, 69117 Heidelberg, Germany \\ e-mail: vanderlaan@iram. fr \\ 2 Institut de Radioastronomie Millimétrique (IRAM), 300 rue de la Piscine, 38406 St. Martin d'Hères, Grenoble, France \\ ${ }^{3}$ European Southern Observatory, Karl-Schwarzschild-Str 2, 85748 Garching, Germany \\ ${ }^{4}$ European Space Agency, Keplerlaan 1, 2200 AG Noordwijk, The Netherlands \\ 5 INAF-Osservatorio Astrofisico di Arcetri, Largo E. Fermi 5, 50125 Firenze, Italy \\ 6 CSIRO Astronomy and Space Science, ATNF, PO Box 76, Epping NSW 1710, Australia \\ 7 Astrophysics Research Institute, Liverpool John Moores University, Birkenhead CH41 1LD, UK \\ 8 Observatoire Astronomique de Strasbourg, CNRS UMR 7550, 67000 Strasbourg, France
}

Received 26 March 2013 / Accepted 17 June 2013

\section{ABSTRACT}

\begin{abstract}
The distribution of gas in the central kiloparsec of a galaxy has a dynamically rapid evolution. Nonaxisymmetries in the gravitational potential of the galactic disk, such as a large scale stellar bar or spiral, can lead to significant radial motion of gaseous material from larger radii to the central region. The large influx of gas and the subsequent star formation keep the central region constantly changing. However, the ability of gas to reach the nucleus proper to fuel an AGN phase is not guaranteed. Gas inflow can be halted at a circumnuclear star forming ring several hundred parsec away. The nearby galaxy NGC 5248 is especially interesting in this sense since it is said to host 2 circumnuclear star forming rings at $100 \mathrm{pc}$ and $370 \mathrm{pc}$ from its quiescent nucleus. Here we present new subarcsecond PdBI+30 m CO(2-1) emission line observations of the central region. For the first time the molecular gas distribution at the smallest stellar ring is resolved into a gas ring, consistent with the presence of a quiescent nucleus. However, the molecular gas shows no ring structure at the larger ring. We combine analyses of the gaseous and stellar content in the central kiloparsec of this galaxy to understand the gas distribution and dynamics of this star forming central region. We discuss the probability of two scenarios leading to the current observations, given our full understanding of this system, and discuss whether there are really two circumnuclear star forming rings in this galaxy.
\end{abstract}

Key words. galaxies: ISM - galaxies: stellar content - galaxies: individual: NGC 5248

\section{Introduction}

The short dynamical times in the central kiloparsec of galaxies lead to rapid changes in the gaseous and stellar distributions there. A large part of this secular evolution is driven by the inflow of new gaseous material from larger radii. The radial motion of gas in disk galaxies is dominated by gravitational torques on all but the smallest scales. These gravitational torques are generated by non-axisymmetric patterns in the disk, such as spiral arms, stellar bars, or ovals. However, continuous gas inflow from the larger scales down to the nucleus proper is not guaranteed under all conditions. For instance, gravitational torques from a large scale stellar pattern become much weaker with decreasing radius due to the increased mass influence of the (symmetric) stellar bulge.

In the case of a large scale stellar bar driving gas inward, the gas distribution is dominated by two spiral arms along the leading sides of the bar. In general, these spirals exist between the inner Lindblad resonance (ILR) and the corotation radius (CR) of the bar (e.g., Huntley et al. 1978; Berman et al. 1979; Athanassoula 1992b). However, gas spirals that are generated

* Full Table 3 and data cube as a FITS file are only available at the CDS via anonymous ftp to cdsarc.u-strasbg. fr (130.79.128.5) or via

http://cdsarc.u-strasbg.fr/viz-bin/qcat?J/A+A/556/A98 by a stellar bar can exist beyond the ILR to the smallest radii to become nuclear spirals (e.g., Pogge \& Martini 2002) under certain conditions. Englmaier \& Shlosman (2000) and Maciejewski (2004b) show that the extent of nuclear spirals mostly depends on the gravitational potential, i.e. the relative strength of the large scale bar and stellar bulge, and the sound speed of the gas. When gas is kinematically warm (high sound speed), the pitch angle of the spiral will be large, resulting in a nuclear spiral which extents towards the nucleus. When gas is kinematically cold (low sound speed), the pitch angle is small, and the spiral will end in a circumnuclear ring. These rings act as reservoirs of gas from larger radii, and, as a result, form stars at a high rate.

When spiral arms end at a circumnuclear ring before reaching the nucleus proper, another mechanism might take over to funnel gas further inward. A nested stellar bar system, where one stellar bar exists within another, is one possible mechanism. This scenario was first proposed by Shlosman et al. (1989), and was followed up by many numerical studies (e.g. Maciejewski et al. 2002; Englmaier \& Shlosman 2004; Maciejewski \& Small 2010). One of the constraints on the stability of a nested stellar bar system is resonance overlap. Even then, gas flow into the region of influence of the inner bar is not always possible, but would be intermittent and dependent on the alignment of the two bars. 

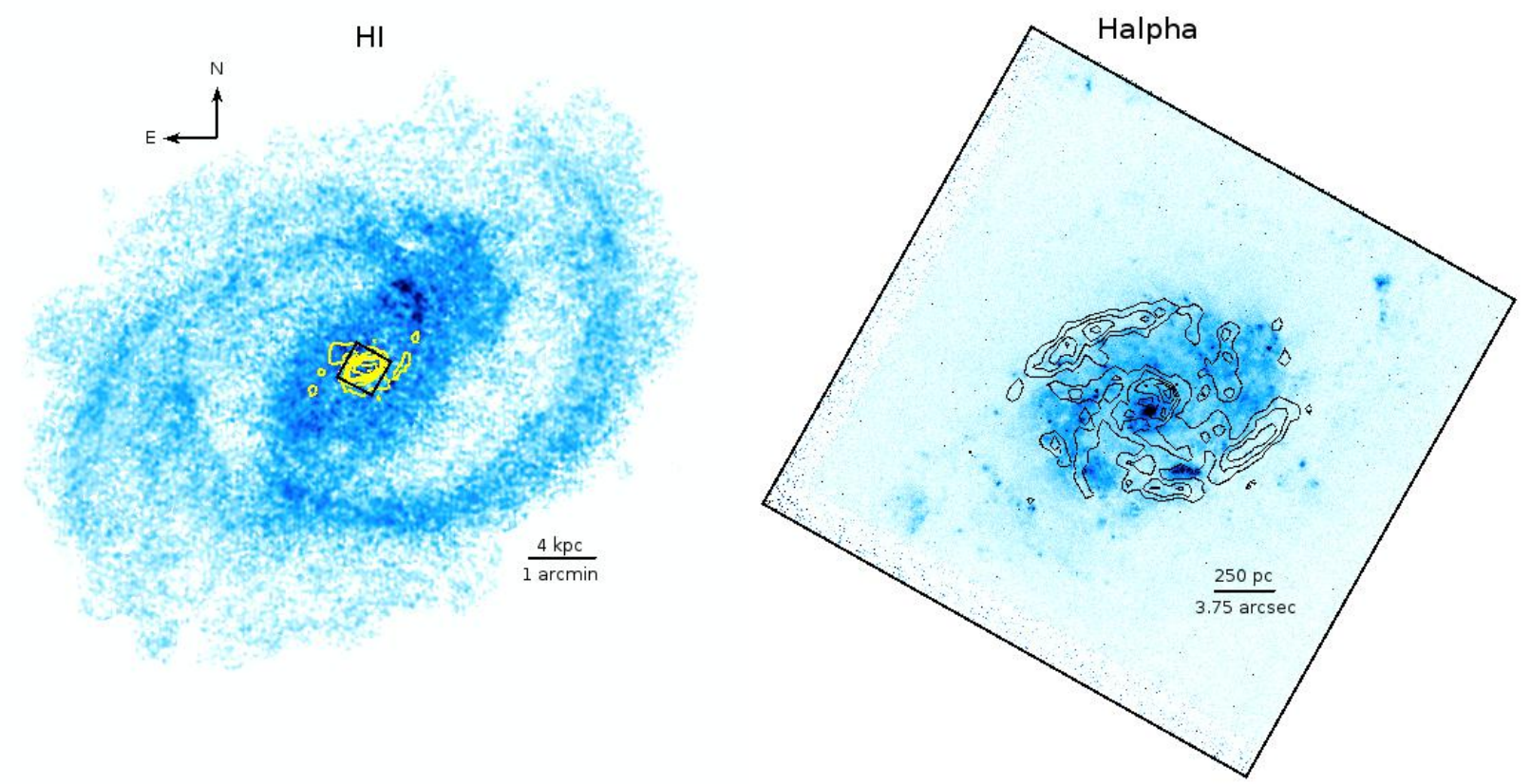

Fig. 1. Indication of the relevant scales in NGC 5248. Left: the observed HI disk (color) goes out to $24 \mathrm{kpc}$. The HI distribution appears dominated by two spiral arms and a large scale bar. BIMA SONG CO(1-0) observations are indicated with yellow contours in this panel. Molecular gas is only detected out to a $3 \mathrm{kpc}$ radius. The molecular gas is distributed in two gas arms and a central concentration. The field of view of the HST $\mathrm{H} \alpha$ map is indicated with a black box. Right: a zoom-in of the HST $\mathrm{H} \alpha$ map. The $\mathrm{H} \alpha$ emission is dominated by the two circumnuclear star forming rings, at $100 \mathrm{pc}$ and $370 \mathrm{pc}$ from the nucleus. The $\mathrm{CO}(2-1)$ observations, presented in Sect. 2.1, are overlaid in black contours.

Nested bars are present in approximately $25 \%$ to $40 \%$ of the population of nearby barred galaxies (e.g. Erwin \& Sparke 2002; Erwin 2004). These secondary bars are much smaller, and their size is no more than $\sim 12 \%$ of the primary bar. Even in nearby galaxies, high angular resolution images are needed to detect them directly.

The NUGA survey (García-Burillo et al. 2003a) has further shown how multiple other patterns might work together to bring gas inward. Multiple configurations seem to be possible, which include lopsided disks (NGC 4826: García-Burillo et al. 2003b; NGC 3718: Krips et al. 2005; NGC 5953: Casasola et al. 2010); bars and spirals (NGC 4569: Boone et al. 2007; NGC 2782: Hunt et al. 2008; NGC 6574: Lindt-Krieg et al. 2008; NGC 4579: García-Burillo et al. 2009; NGC 6951: Van der Laan et al. 2011), and rings (NGC 7217: Combes et al. 2004; NGC 3147: Casasola et al. 2008; NGC 1961: Combes et al. 2009).

Finally, for smaller rings, dynamical friction or viscose torques may lead to further gas flow towards the nucleus (Combes 2008). As discussed in García-Burillo et al. (2005), the timescales for viscose torques only become comparable to those of gravitational torques at $100-200 \mathrm{pc}$ scales when strong density contracts (e.g. gas rings) are present.

When no secondary pattern is present, or the ring is at too large radius for viscose torques to be effective, and a circumnuclear star forming ring forms, the ring is an effective barrier to further gas inflow (Athanassoula 2000; Maciejewski 2004b; Haan et al. 2009; Van der Laan et al. 2011).

In this paper, we study the case of the nearby barred galaxy NGC 5248. This galaxy is said to host two circumnuclear star forming rings at $1.5^{\prime \prime}(100 \mathrm{pc})$ and $6^{\prime \prime}(370 \mathrm{pc})$ from its quiescent nucleus, in apparent contradiction with circumnuclear rings being effective gas barriers. Atomic and molecular gas has been detected at radii of less than $1.5^{\prime \prime}$ from the nucleus. NGC 5248 is a nearby $\mathrm{SAB}(\mathrm{rs}) \mathrm{bc}$ galaxy at a distance of $12.7 \mathrm{Mpc}$
Table 1. Global properties of NGC 5248.

\begin{tabular}{lcc}
\hline \hline Parameter & Value & Reference \\
\hline Type & $\mathrm{SAB}(\mathrm{rs}) \mathrm{bc}$ & $(1)$ \\
Dynamic center & & \\
$\quad$ RA $(\mathrm{J} 2000)$ & $13^{\mathrm{h}} 37^{\mathrm{m}} 32.0^{\mathrm{s}}$ & $(2,3)$ \\
Dec $(\mathrm{J} 2000)$ & $08^{\circ} 53^{\prime} 06.74^{\prime \prime}$ & $(2,3)$ \\
Inclination angle & $43.1^{\circ}$ & $(3,4)$ \\
Position angle & $115^{\circ}$ & $(3,4)$ \\
Adopted distance & $12.7 \mathrm{Mpc}$ & $(5)$ \\
Systemic velocity & $1153 \mathrm{~km} \mathrm{~s}^{-1}$ & $(3,4)$ \\
Mass $\mathrm{H}_{2}(r<1.2 \mathrm{kpc})$ & $4.0 \times 10^{8} M_{\odot}$ & $(3)$ \\
Mass $\mathrm{H}_{2}(r<300 \mathrm{pc})$ & $1.0 \times 10^{8} M_{\odot}$ & $(3)$ \\
\hline
\end{tabular}

References. (1) de Vaucouleurs et al. (1991); (2) NED; (3) this work; (4) Haan et al. (2008); (5) Tully et al. (2009); (6) Jogee et al. (2002a).

(Tully et al. 2009), i.e. a spatial scale of $1^{\prime \prime}=61.6 \mathrm{pc}$. Its global properties are listed in Table 1.

Spiraling dust lanes between $6^{\prime \prime}(370 \mathrm{pc})$ and $90^{\prime \prime}(5.5 \mathrm{kpc})$ radius are seen in broad-band $B$ and $I-K$ images (Figs. 2 and 4a of Jogee et al. 2002b). The $\mathrm{CO}(1-0)$ distribution mapped by the BIMA SONG survey (Helfer et al. 2003) also shows some lowlevel CO emission at these positions (Fig. 1, left panel). Jogee et al. (2002a) detected a large scale stellar bar in this galaxy, with a semi-major axis of $95^{\prime \prime}(5.9 \mathrm{kpc})$, a PA of $135^{\circ}$, and a deprojected ellipticity of 0.44 . Its pattern speed is approximated at $30 \mathrm{~km} \mathrm{~s}^{-1} \mathrm{kpc}$.

The two circumnuclear star forming rings are detected in $\mathrm{H} \alpha$ emission (Fig. 1, right panel, Laine et al. 2001; Maoz et al. $2001)$. The outer one at $6^{\prime \prime}(370 \mathrm{pc})$ is very broad, with a radiusto-width ratio of $\sim 2$. The molecular gas distribution, as traced by $\mathrm{CO}(1-0)$ shows two spiral arms that are co-spatial with (part of) this circumnuclear ring. The orientation of these molecular spiral 
arms is similar to the larger dust spiral arms. In fact, Jogee et al. (2002b) argue that both molecular gas, stars and dust spiral arms trace the same density wave, that thus winds up over $2 \pi$, from the outer edge of the large scale bar, past the larger circumnuclear ring, to the very center/smaller circumnuclear ring. The inner circumnuclear $\mathrm{H} \alpha$ ring at $1.5^{\prime \prime}(100 \mathrm{pc})$ is narrow and sharply defined. In the circumnuclear region all $\mathrm{H} \alpha$ emission arises from these two rings, with a void between the two rings.

The primary goal of this work is to understand the makeup of the central $1.5 \mathrm{kpc}$ in this galaxy. We investigate the gas motions, and determine the stellar ages, to understand the gas distribution and dynamics, as well as the formation of the two circumnuclear star forming rings. To this end, new interferometric observations in the ${ }^{12} \mathrm{CO}(2-1)$ line transition are presented in Sect. 2. These observations have subarcsecond spatial resolution and resolve the inner circumnuclear ring in molecular gas for the first time. In Sect. 3, gas morphology and non-circular motions in the gas are determined using archival $\mathrm{HI}$ and $\mathrm{CO}(1-0)$ data, together with our new $\mathrm{CO}(2-1)$ observations. In Sect. 4 a thorough investigation of the ages of the stars in both rings is made. Work by Maoz et al. (2001) has identified and age dated star clusters observed from HST optical images before, but we repeat the age dating of the star clusters with updated stellar evolution models and our own $\chi^{2}$ fitting script. A SAURON IFU data set (Dumas et al. 2007) is added to analyze the overall contribution of young stars in the central kiloparsec. In Sect. 5 we discuss the star formation (history) and the gas dynamics in and between these two rings and discuss two possible scenarios that connect the different components. We summarize in Sect. 6.

\section{Observations and data reduction}

\subsection{IRAM PdBI+30M CO(2-1) data}

IRAM PdBI observations in ABCD configuration were carried out between January 2009 and December 2010 using the full 6-antenna array. The correlator was centered at $229.6547 \mathrm{GHz}$, corresponding to a heliocentric velocity of $1153 \mathrm{~km} \mathrm{~s}^{-1}$, to observe the redshifted ${ }^{12} \mathrm{CO}(2-1)$ emission line. Quasar $3 \mathrm{C} 273$ and MWC 349 were used for flux calibration. During the observations in D-array, the WIDEX correlator was working in parallel with the narrow-band correlator, and simultaneously recorded the full $3.6 \mathrm{GHz}$ bandwidth. All observations were reduced using standard routines in the GILDAS/CLIC software package ${ }^{1}$. A free addition to the $\mathrm{CO}$ observations with the WIDEX correlator at the PdBI is a $1 \mathrm{~mm}$ continuum map. The WIDEX data were integrated over their full spectral range, excluding the $\mathrm{CO}(2-1)$ emission line region. The so obtained $1 \mathrm{~mm}$ continuum map (Fig. 2) has $128 \times 128$ pixels, a pixel scale of $0.3^{\prime \prime} /$ pixel, a beam size of $2.4^{\prime \prime}$ by $1.3^{\prime \prime}$, and a PA of $18.8^{\circ}$. The highest emission peak reaches a $13 \sigma\left(1 \sigma=1.8 \mathrm{mJy}^{-1}\right.$ beam $\left.^{-1}\right)$ intensity. The $1 \mathrm{~mm}$ distribution will be discussed in Sect. 5.1.

Single-dish observations for the central $44^{\prime \prime}(2.7 \mathrm{kpc})$ by $44^{\prime \prime}(2.7 \mathrm{kpc})$ were obtained with the IRAM $30 \mathrm{~m}$ telescope on July 13 and 14, 2011. The $1 \mathrm{~mm}$ receivers where tuned to $229.6553 \mathrm{GHz}$. A bandwidth of $640 \mathrm{MHz}$ was covered by 512 channels with a width of $1.25 \mathrm{MHz}\left(2.6 \mathrm{~km} \mathrm{~s}^{-1}\right)$ at $1 \mathrm{~mm}$. The spacing between individual grid points was $4^{\prime \prime}$. Typical system temperatures during the observations were $360 \mathrm{~K}$ for the $1 \mathrm{~mm}$ receivers. The data reduction was done using the GILDAS/CLASS software package.

The single-dish observations were used to compute the short spacing correction (SSC) and recover the large-scale low-level

1 http://www.iram.fr/IRAMFR/GILDAS

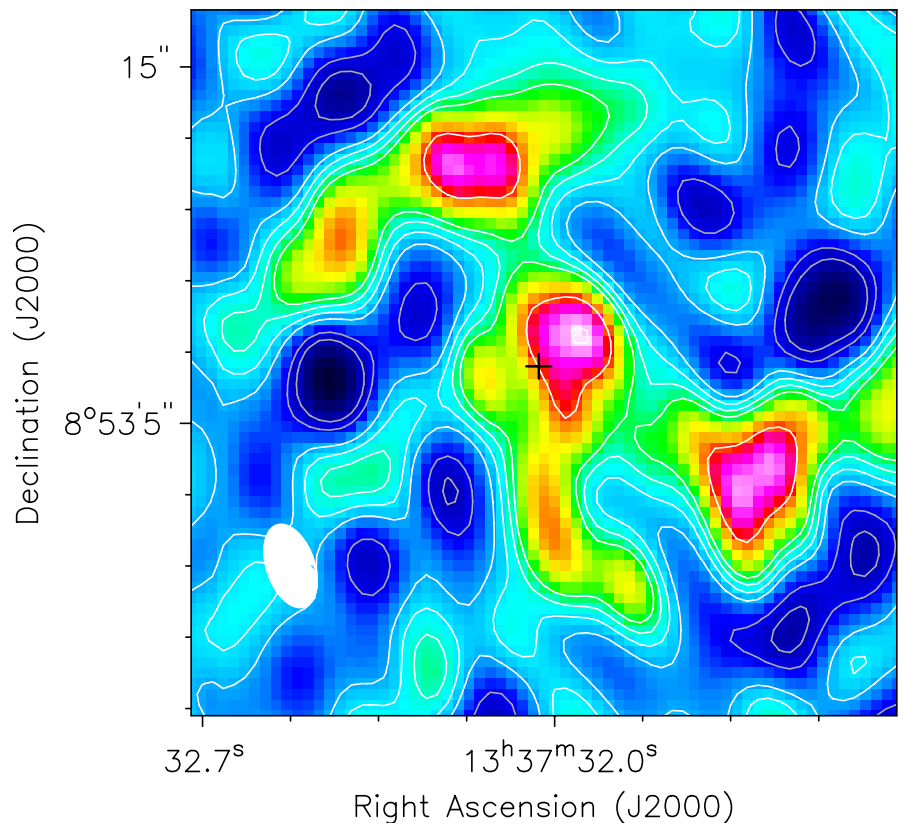

Fig. 2. $1 \mathrm{~mm}$ continuum as observed with the PdBI Widex instrument. The telescope was positioned in $\mathrm{D}$ configuration. Contours give the $-3 \sigma,-2 \sigma, 0,2 \sigma, 3 \sigma$ and then multiples of $5 \sigma$ flux levels $(1 \sigma=$ $1.8 \mathrm{mJy} / \mathrm{beam})$. The galaxy center is indicated by a black cross. The beam size is $2.4^{\prime \prime} \times 1.3^{\prime \prime}$ and is indicated in the bottom-left corner. Pixel scale is $0.30^{\prime \prime}$. A color version of this figure is available in the electronic version.

flux for the $\mathrm{CO}(2-1)$ line. The $30 \mathrm{~m}$ observations were reprojected to the field center and frequency of the PdBI observations. The bandwidth coverage of the $30 \mathrm{~m}$ data was resampled to match the velocity axis of the interferometric observations. A combined data cube was produced using the task "UV-short" in GILDAS.

A cleaned SSC image cube was produced using uniform weighting with the GILDAS/MAPPING software package. The beam size/resolution of the uniform weighted $\mathrm{CO}(2-1)$ data is given in Table 2, as well as the rms noise per channel. The image cube has 1024 by 1024 pixels and 121 velocity channels, with a pixel scale of $0.1^{\prime \prime} /$ pixel and velocity bins of $5 \mathrm{~km} \mathrm{~s}^{-1}$. CLEANing was done down to the $2 \sigma$ noise level with the assistance of CLEAN polygon regions defined individually for each channel with line emission present.

The channel maps of the cleaned image cube are presented in Fig. 3. $\mathrm{CO}(2-1)$ emission is seen in the channels $[-130: 145] \mathrm{km} \mathrm{s}^{-1}$ relative to the $1153 \mathrm{~km} \mathrm{~s}^{-1}$ systemic velocity. In the individual channels the emission is extended from northeast to south-west, in accordance with the position angle of $115^{\circ}$ for this galaxy (see Table 1). The dirty beam is extended northsouth due to the low declination of the source, which prevents a more circular population of the $(u, v)$-plane. The final clean beam has the size $0.62^{\prime \prime} \times 0.34^{\prime \prime}$, with a PA of $27.3^{\circ}$.

\subsection{Ancillary archival data}

For this study we also make use of the following data sets that have been presented in the literature before.

\subsubsection{SAURON IFU data}

NGC 5248 was observed with the SAURON instrument (Bacon et al. 2001) at the William Herschel Telescope in March 2004 


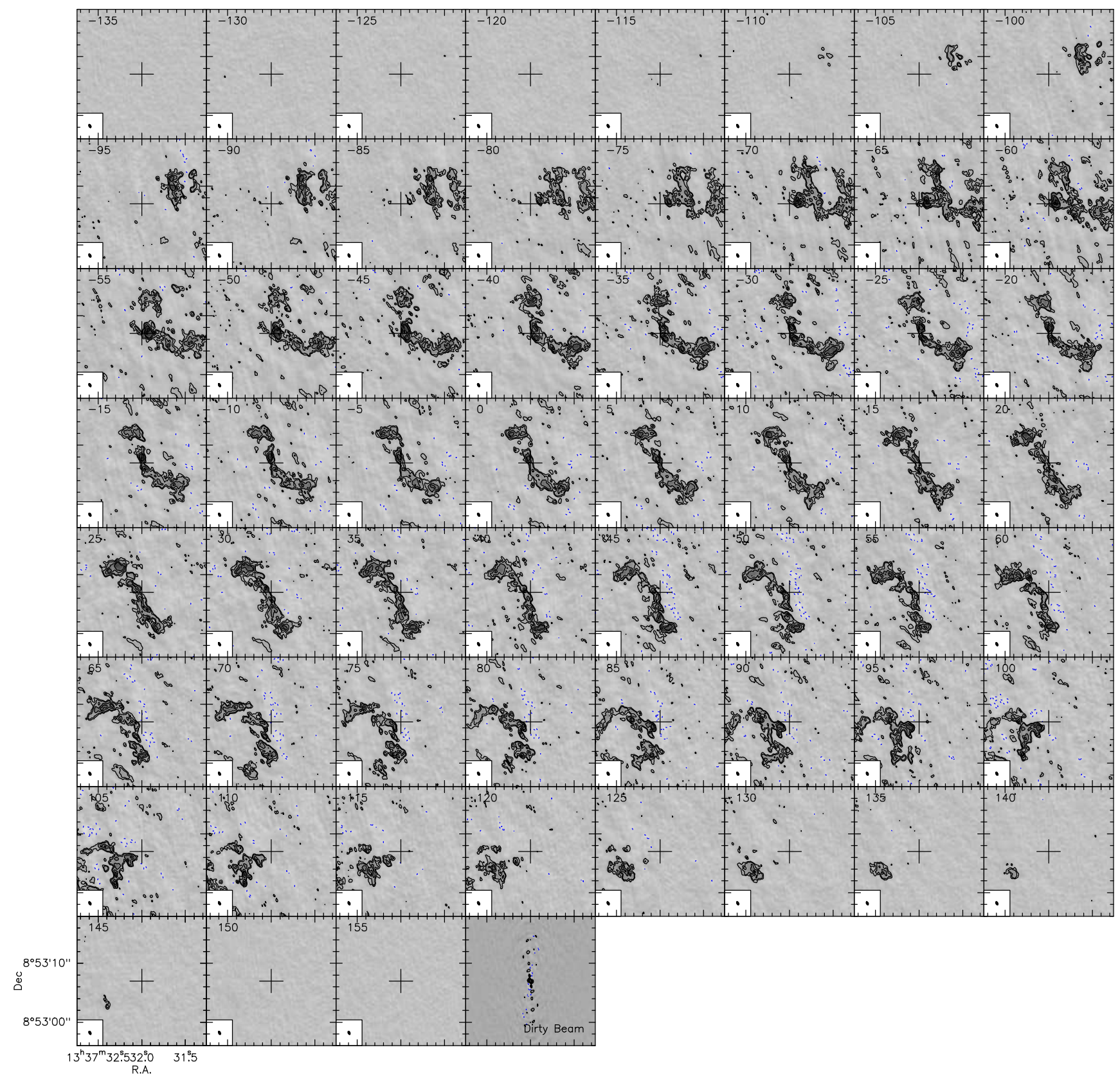

Fig. 3. Channel maps of the uniform weighted ${ }^{12} \mathrm{CO}(2-1)$ SSC image cube. The size of the displayed channel maps is $22^{\prime \prime}$ by $22^{\prime \prime}$, which is the size of the primary beam of the PdBI observations. The contours are at $-3 \sigma, 3 \sigma, 5 \sigma, 10 \sigma, 15 \sigma$ and $25 \sigma$, with $1 \sigma=2.8 \mathrm{mJy}^{-1}$ beam ${ }^{-1}$. The velocity relative to the systemic velocity of the galaxy $\left(v_{\text {sys }}=1153 \mathrm{~km} \mathrm{~s}^{-1}\right)$ is indicated in the upper left corner. The phase center of the observations is indicated by a cross in each channel map. The clean beam $\left(0.62^{\prime \prime}\right.$ by $\left.0.34^{\prime \prime}\right)$ is shown in the lower left corner of each channel map and the dirty beam is shown in the lower right panel.

Table 2. Key numbers of the $\mathrm{CO}(2-1)$ and $1 \mathrm{~mm}$ continuum data.

\begin{tabular}{lccc}
\hline \hline & $\begin{array}{c}\text { Beam size } \\
\left({ }^{\prime \prime} \times{ }^{\prime \prime}\right)\end{array}$ & $\begin{array}{c}\text { PA } \\
\left({ }^{\circ}\right)\end{array}$ & $\begin{array}{c}\mathrm{rms} \\
(\mathrm{mJy} / \text { beam })\end{array}$ \\
\hline $\mathrm{CO}(2-1)$ & $0.62 \times 0.34$ & 27.3 & 2.8 \\
$1 \mathrm{~mm}$ cont. & $2.4 \times 1.3$ & 18.8 & 1.8 \\
\hline
\end{tabular}

Notes. The $\mathrm{CO}(2-1)$ cube is constructed from PdBI ABCD $+30 \mathrm{~m}$ observations, the $1 \mathrm{~mm}$ cube only from the PdBI D configuration observations. Both cubes are imaged with uniform weighting.

and maps were first presented by Dumas et al. (2007). The field of view (FoV) of the SAURON integral field unit (IFU) instrument is $33^{\prime \prime}(2.0 \mathrm{kpc}) \times 41^{\prime \prime}(2.5 \mathrm{kpc})$ with square $0.94^{\prime \prime}$ lenses. The final data cube is built out of 5 individual $30 \mathrm{~min}$ exposures and has $0.8^{\prime \prime} \times 0.8^{\prime \prime}$ spaxels. The spectral range covered is 4825-5280 $\AA$, with a $4.2 \AA$ resolution and spectral sampling of $1.15 \AA$. Data reduction and flux calibration were done with the dedicated XSauron software and is explained in detail in Dumas et al. (2007). Vonoroi binning was applied to increase the signalto-noise ratio $(\mathrm{S} / \mathrm{N})$ to 40 . For the central $\sim 9^{\prime \prime}(0.5 \mathrm{kpc})$ radius the resulting Vonoroi bins are equal to the original spaxels (the $\mathrm{S} / \mathrm{N}$ was already above 40 ).

The SAURON spectral range (4825-5280) $\AA$ covers the stellar absorption lines $\mathrm{H} \beta, \mathrm{Fe} 5015, \mathrm{Mg} \beta$, and $\mathrm{Fe} 5270$, as well as the gaseous emission line $\mathrm{H} \beta$, and the doublets 

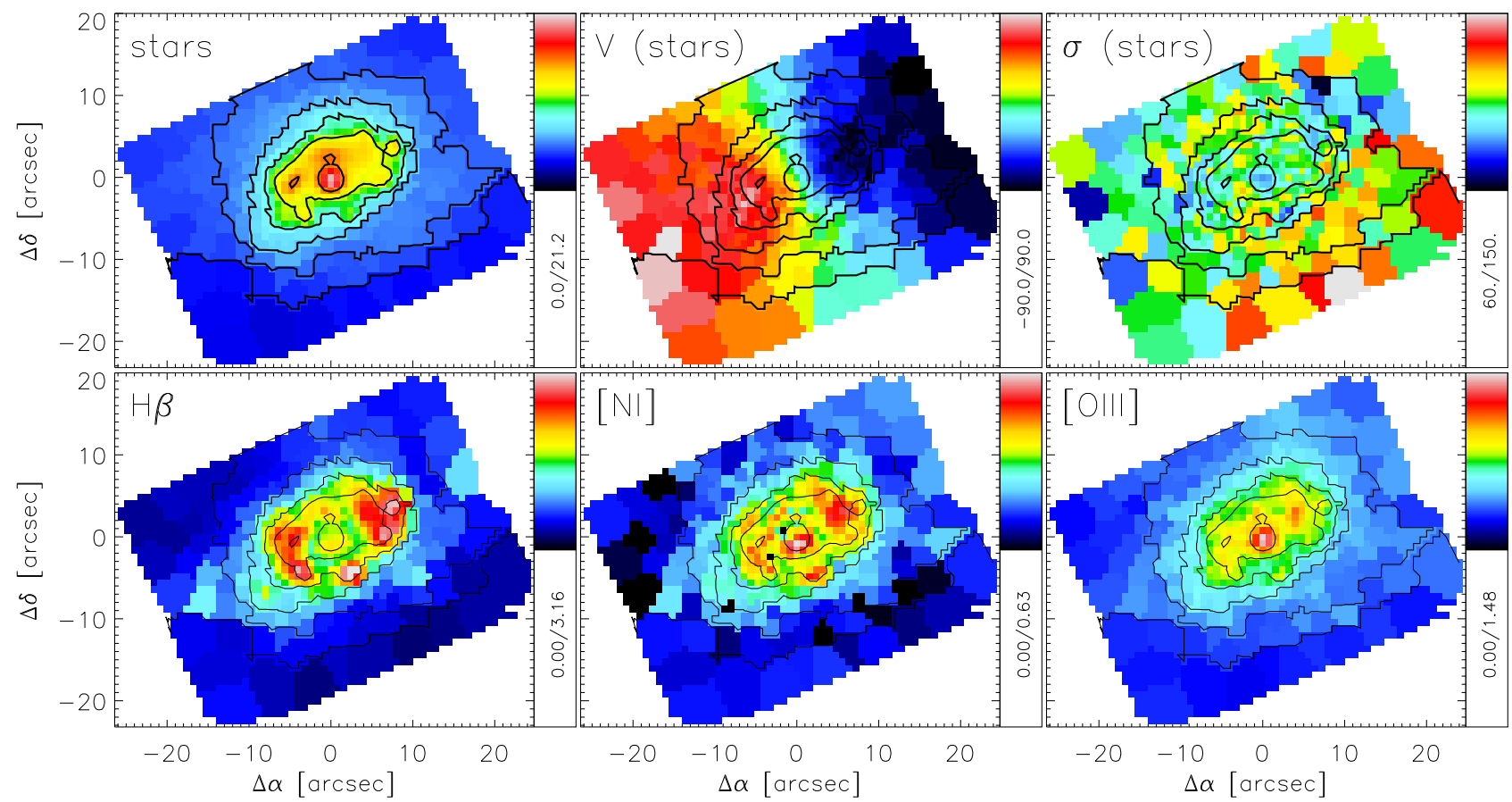

Fig. 4. Top: SAURON maps of the stellar continuum, velocity field and velocity dispersion. Bottom: intensity distributions of the emission lines in the SAURON spectral range; $\mathrm{H} \beta$, [NI] and [OIII]. The maps are oriented with north pointing up, and east to the left. The contours indicate the stellar flux in all panels and are included to guide the eye. A color version of this figure is available in the electronic version.

$[\mathrm{OIII}](\lambda(4959,5007) \AA)$, and $[\mathrm{NI}](\lambda(5200,5202) \AA)$. Each spaxel is fitted with a combination of single-stellar population spectra (SSP) from the MILES library² (Sánchez-Blázquez et al. 2006; Falcón-Barroso et al. 2011) and Gaussian emission line profiles using the IDL-based routines pPXF (Cappellari \& Emsellem 2004; Emsellem et al. 2004) and GANDALF (Sarzi et al. 2006). The kinematics of the emission lines were fixed to the [OIII] doublet. The combined use of pPXF and GANDALF takes into account the fact that $\mathrm{H} \beta$ is present both in absorption and emission.

Several of the resulting maps are shown in Fig. 4. The top row in this figure shows the stellar continuum, stellar velocity field and stellar velocity dispersion. The distributions of the emission lines $\mathrm{H} \beta$, [NI], and [OIII] are shown on the bottom row. To guide the eye, stellar flux contours are overlaid on all panels. The nebular emission lines are strongest in the outer circumnuclear ring, which is most clearly traced in $\mathrm{H} \beta$ emission, but also in [NI] and [OIII]. Some $\mathrm{H} \beta$ emission is also seen from the inner $2^{\prime \prime}$, and seems to be not located at the nucleus, but at the inner circumnuclear ring. The [NI] line emission peaks at this same position at the inner ring. The ratio $[\mathrm{NI}] / \mathrm{H} \beta$ is an indicator of the intensity of the continuum fluorescent excitation connected to HII regions (Bautista 1999; Sarzi et al. 2010; Ferland et al. 2012). Its value $(\sim 0.1)$ in the southern half of the inner ring implies recent, intense star formation. In the outer circumnuclear ring the positions of highest $\mathrm{H} \beta$ and [NI] line emission intensity also are co-spatial, but the $[\mathrm{NI}] / \mathrm{H} \beta$ ratio is much lower $(\sim 0.03)$. The lower value would indicate that recent star formation is less intense in the outer ring. At the outer circumnuclear ring the emission of all three lines is strongest at the north-west and south-east quadrants of the ring. This could hint at an azimuthal star formation distribution. The stellar continuum does not appear particularly correlated with the emission line intensities, which suggests that the distribution of stellar

\footnotetext{
2 http://miles.iac.es
}

light is not overall dominated by the youngest stellar population. Stellar continuum emission is higher at the northern section of the ring, than at the southern part.

\subsection{2. $\mathrm{HI}$}

NGC 5248 was observed with the VLA in C and D array as part of a larger campaign (Haan et al. 2008, 2009) in 2003 and 2004. We refer to that work for details of the observations and data reduction. The robust weighted data cube used in this work has a spatial resolution of $20^{\prime \prime}$, with a $2^{\prime \prime}$ pixel size and a FoV of $34^{\prime}(125 \mathrm{kpc}) \times 34^{\prime}(125 \mathrm{kpc})$. The total HI flux measured in these observations is $81.3 \mathrm{Jy} \mathrm{km} \mathrm{s}^{-1}$, which corresponds to an atomic gas mass of $4.32 \times 10^{9} M_{\odot}$ (Haan et al. 2008).

The HI distribution (Fig. 1, left panel) is dominated by a central bar-like structure (most likely a response to the $95^{\prime \prime}(5.9 \mathrm{kpc})$ large scale stellar bar) and spiral arms. The HI intensity is somewhat higher within the central region, but within that there is little to no variation, nor is there an intensity peak towards the center. The morphology of the atomic gas does not trace spiral structure within the central region, except for a partial spiral arm in the north-west that continues into a $\mathrm{CO}$ arm at smaller radii.

\subsubsection{BIMA SONG $\mathrm{CO}(1-0)$}

The BIMA SONG survey (Helfer et al. 2003) was designed to systematically observe the $\mathrm{CO}(1-0)$ distribution in 44 nearby galaxies. NGC 5248 was observed in May 1999. The total FoV of these data are 194" $(12 \mathrm{kpc}) \times 194^{\prime \prime}(12 \mathrm{kpc})$ the data have a resolution of $6^{\prime \prime}$, and are short-spacing corrected. The resulting data cube and moment maps are publicly available from $\mathrm{NED}^{3}$. A contour plot of the intensity distribution is included in Fig. 1 (left panel).

\footnotetext{
3 NASA/IPAC Extragalactic Database.
} 
The BIMA SONG intensity distribution shows that the molecular gas density, as traced by the $\mathrm{CO}(1-0)$ flux intensity, increases towards the center, with an "S"-like shape, which is resolved into two spiral arms in the higher angular resolution $\mathrm{CO}(1-0)$ maps by Jogee et al. (2002b). The main $\mathrm{CO}(1-0)$ emission structure extends out to $\sim 22^{\prime \prime}(1.4 \mathrm{kpc})$ from the nucleus. At larger radii, two spiral arms are partly traced by the $\mathrm{CO}(1-0)$ emission. No $\mathrm{CO}(1-0)$ line emission is detected outside a $3 \mathrm{kpc}$ radius.

\subsubsection{HST}

In Maoz et al. (2001) a thorough detection of star clusters in the circumnuclear rings of NGC 5248 is presented. This work was based on HST observations in 5 filters; F220W, F336W, F547M, $F 814 \mathrm{~W}$ and $F 160 \mathrm{~W}$. A table with the observed fluxes for each star cluster in each band was available for download with the paper. We refer to that work for details on the data reduction and cluster finding. In total fluxes were derived for 507 star cluster candidates (i.e. point sources) in one or more filter bands. The radial distribution of candidates in the outer circumnuclear ring is wide, $\Delta r \sim 2^{\prime \prime}$, especially in the north-west region of the outer ring. The distribution at the inner ring is very narrow, $\Delta r \leq 0.2^{\prime \prime}$.

\section{Molecular gas in the center of NGC 5248}

The existence of two circumnuclear star formation rings in NGC 5248 is unexpected in relation to the understanding that circumnuclear rings are effective gas barriers. Especially since this galaxy is believed to have only one large scale stellar bar. Fortunately, the morphology and kinematics of the gas in the circumnuclear region can be used to show the (formation) relation between the two rings, if any, and other structures in the circumnuclear region.

\section{1. $\mathrm{CO}(2-1)$ morphology}

The new $\mathrm{CO}(2-1)$ observations presented here have subarcsecond resolution and offer an unprecedented view of the molecular gas in the central region of NGC 5248. The intensity distribution of the $\mathrm{CO}(2-1)$ line emitting gas is shown in Fig. 5. At 6" $(370 \mathrm{pc})$ from the nucleus two partial spiral arms are seen in the north and south. The location of the spiral arms is cospatial with the location of the outer circumnuclear star forming ring. Inside that, the morphology shown in Fig. 5 is difficult to interpret. It can be explained as two continuing spirals that extend the two outer $\mathrm{CO}$ arms inwards for another $180^{\circ}$, or as two additional, fractured, lower-intensity arms originating east and west of the nucleus. At $1.5^{\prime \prime}(100 \mathrm{pc})$ radius from the center we find a nearly-full ring. This location is consistent with the location of the inner circumnuclear star forming ring, and it is the first time that this inner ring is resolved in molecular gas. The $\mathrm{CO}(2-1)$ intensity reaches the highest values in this ring. Very little $\mathrm{CO}(2-1)$ emission is detected from the nucleus.

An integrated flux of $684 \mathrm{Jy} \mathrm{km} \mathrm{s}^{-1}$ is measured within the central $10^{\prime \prime}$ radius, of which $177 \mathrm{Jy} \mathrm{km} \mathrm{s}^{-1}(\sim 26 \%)$ are within the central $3^{\prime \prime}$ radius (the inner ring). This corresponds to a molecular gas mass of $4.0 \times 10^{8} M_{\odot}$ and $1.0 \times 10^{8} M_{\odot}$, respectively. These values are corrected for the helium abundance, and an assumed, constant, $I_{\mathrm{CO}(2-1)} / I_{\mathrm{CO}(1-0)}$ line ratio of 0.89 (Braine et al. 1993) was used. Similar ratios are found for the central regions of other nearby galaxies, (Koda et al. 2012; Sandstrom et al. 2012). The Galactic $X_{C O}$ value

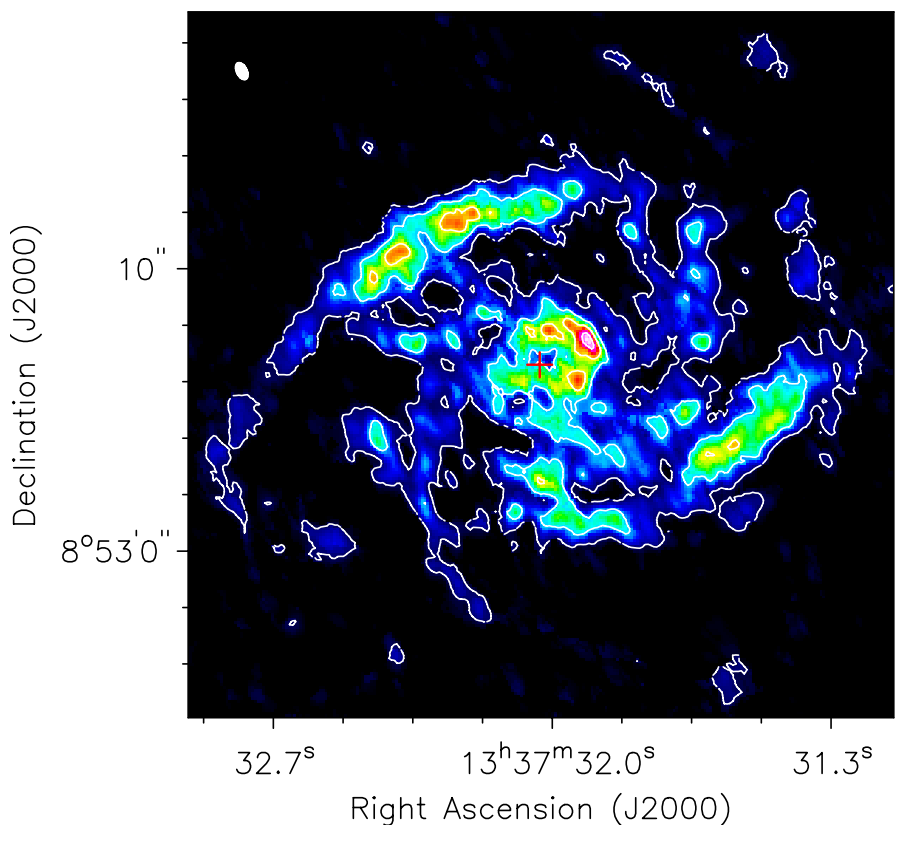

Fig. 5. Integrated $\mathrm{CO}(2-1)$ emission in uniform weighting for the inner $1.5 \mathrm{kpc}$ radius of NGC 5248. The $\mathrm{CO}(2-1)$ emission has been integrated from $-135 \mathrm{~km} \mathrm{~s}^{-1}$ to $155 \mathrm{~km} \mathrm{~s}^{-1}$ relative to the systemic velocity of $1153 \mathrm{~km} \mathrm{~s}^{-1}$. Contours run from $5 \sigma$ in $10 \sigma$ steps $(1 \sigma=$ $0.014 \mathrm{Jy}_{\text {beam }}^{-1} \mathrm{~km} \mathrm{~s}^{-1}$ ) The red cross indicates the position of the dynamic center. The beam size is shown in the upper left corner and corresponds to the values listed in Table 2. A color version of this figure is available in the electronic version.

of $2.2 \mathrm{e} 20 \mathrm{~cm}^{-2}\left[\mathrm{~K} \mathrm{~km} \mathrm{~s}^{-1}\right]^{-1}$ is also assumed ${ }^{4}$. Table 1 summarizes these and other relevant numbers for NGC 5248.

Comparison between the $\mathrm{CO}(2-1)$ emitting gas distribution presented here and the $\mathrm{CO}(1-0)$ distribution presented in Jogee et al. (2002b) shows that $\mathrm{CO}(1-0)$ and $\mathrm{CO}(2-1)$ emission is both present in the two spiral arms that trace the outer circumnuclear star forming ring in the north and south. Inside the outer ring radius the two line emission distributions differ. The $\mathrm{CO}(1-0)$ emission flux is lower than in the spiral arms, while the $\mathrm{CO}(2-1)$ emission is brightest in this region. This could indicate that $\mathrm{CO}(2-1)$ is preferentially excited over $\mathrm{CO}(1-0)$ at these smaller radii (this would imply a higher $\mathrm{I}_{\mathrm{CO}(2-1)} / \mathrm{I}_{\mathrm{CO}(1-0)}$ ratio for the inner ring, e.g. Armour \& Ballantyne 2012, and decrease the molecular gas mass in the inner ring with respect to the outer ring). The morphology of the $\mathrm{CO}(1-0)$ and $\mathrm{CO}(2-1)$ emission within the outer ring radius is also different. The $\mathrm{CO}(1-0)$ emission is distributed in an elongated structure parallel to the $\mathrm{CO}$ spiral arms that are cospatial with the outer ring, with emission peaks at the inner circumnuclear ring (east and west) and extending low-level emission spurs at the outer circumnuclear ring raidus, whereas the $\mathrm{CO}(2-1)$ emission shows the low intensity spiral arms in the east and west at the outer ring radius and the distinct structure of the inner circumnuclear ring in the center. The different appearance in $\mathrm{CO}(1-0)$ and $\mathrm{CO}(2-1)$ line emission inside the large star forming ring could reflect changes in the excitation conditions from the outer to the inner ring, with young star formation, i.e. heating of the molecular gas, being a very likely explanation.

4 Recent work by Sandstrom et al. (2012) seems to indicate that this value is actually lower in the centers of galaxies. A ratio lower by a factor $x$, would lower the derived gas mass by a factor $x$. 

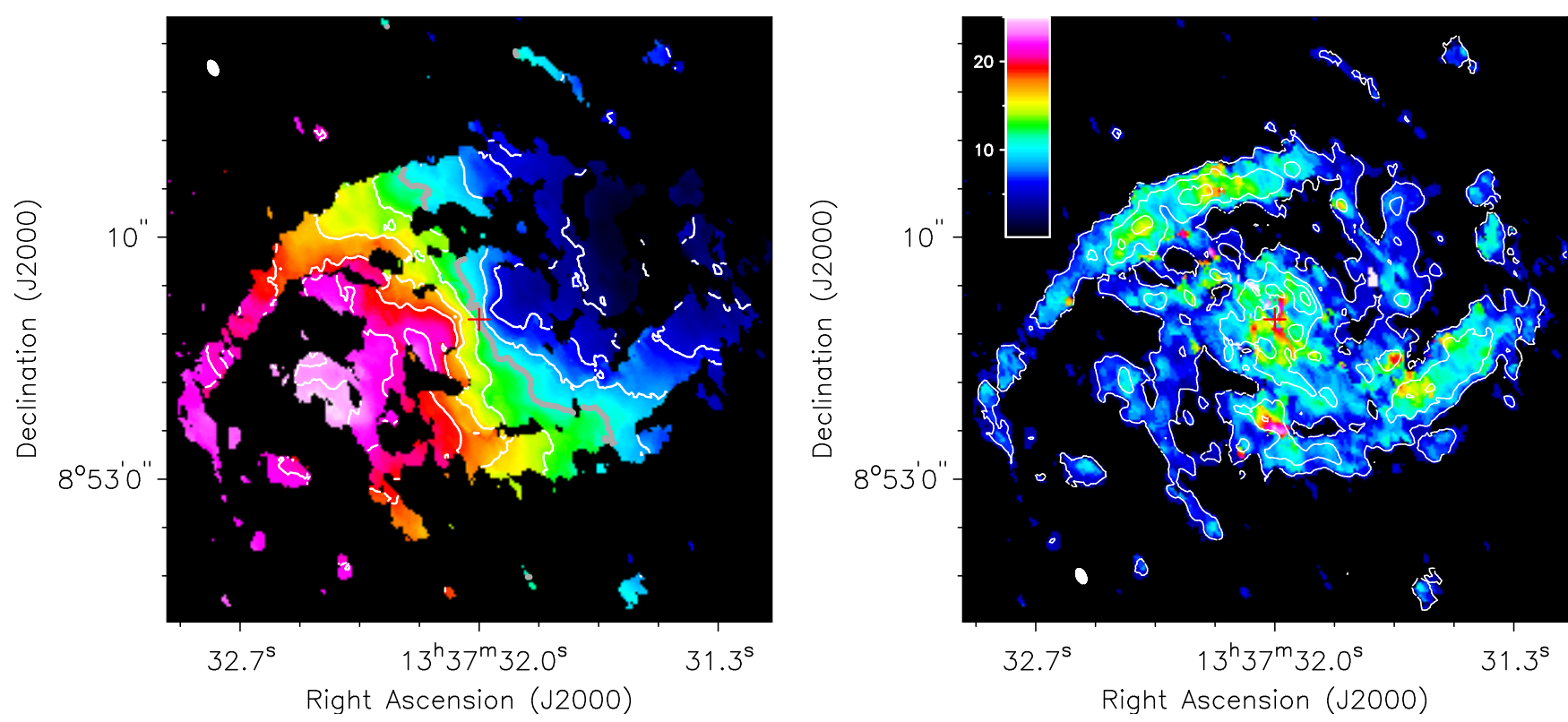

Fig. 6. Left: first moment map of the $\mathrm{CO}(2-1)$ emission. Contours: -150 to $150 \mathrm{~km} \mathrm{~s}^{-1}$ with $25 \mathrm{~km} \mathrm{~s}^{-1}$ steps, $0 \mathrm{~km} \mathrm{~s}^{-1}$ thick gray contour. Velocities are relative to the systemic velocity, $1153 \mathrm{~km} \mathrm{~s}^{-1}$, with negative velocities in blue and positive velocities in red. Right: second moment map of the $\mathrm{CO}(2-1)$ emission. The color bar shows the dispersion in $\mathrm{km} \mathrm{s}^{-1}$. Contours are the integrated $\mathrm{CO}(2-1)$ emission in $10 \sigma$ steps starting at $5 \sigma$ $\left(1 \sigma=0.014 \mathrm{Jy}_{\text {beam }}^{-1} \mathrm{~km} \mathrm{~s}^{-1}\right)$. The dynamic center is given with a red cross in both panels. A color version of this figure is available in the electronic version.

Recall that in the SAURON data (Sect. 2.2.1) a high $[\mathrm{NI}] / \mathrm{H} \beta$ ratio was seen at the inner ring, but not the outer ring.

\section{2. $\mathrm{CO}(2-1)$ kinematics}

The first (velocity field) and second (velocity dispersion) moment maps of the $\mathrm{CO}(2-1)$ data are shown in Fig. 6. The velocity field of the $\mathrm{CO}(2-1)$ emitting gas is dominated by circular velocity; it shows the "spider diagram" contours of an inclined rotating disk. The dynamical center is equal to the photometric center, within the observational resolution. The kinematic axis of the velocity field of the $\mathrm{CO}(2-1)$ emitting gas and the stellar velocity field of the SAURON observations are very similar. The $\mathrm{CO}(2-1)$ velocity range is broader than the SAURON stellar velocity range, but the velocity dispersion is broader in the stars. This implies that the molecular gas is kinematically colder $\left(\sigma_{\mathrm{CO}(2-1)} \sim 5-20 \mathrm{~km} \mathrm{~s}^{-1}\right)$ than the stars, and shows more ordered motion. There is no offset between the stellar and gaseous disk.

Given that spiral arms are usually found at the leading side of a bar and that the blue-shifted velocities are in the west, NGC 5248 must be rotating clockwise. This implies that the south-western part of NGC 5248 is the near side of the galaxy, and the north-eastern part the far side. The orientation of the disk is important to be able to determine later on whether non-circular motions imply in- or out-flows.

The highest $\mathrm{CO}(2-1)$ velocity dispersions $\left(15-20 \mathrm{~km} \mathrm{~s}^{-1}\right)$ are seen in the spiral arms at $6^{\prime \prime}(370 \mathrm{pc})$ from the nucleus and inside the inner $4^{\prime \prime}(250 \mathrm{pc})$, i.e. the inner ring region. Given the location, the higher velocity dispersions could be indicative of either enhanced turbulence due to recent star formation or noncircular motions caused by shocks or shear in the gas.

As a first investigation of the non-circular motion two position-velocity (pv) diagrams were extracted from the $\mathrm{CO}(2-1)$ data cube; one along the major kinematic axis $\left(\mathrm{PA} 115^{\circ}\right)$ and one along the minor kinematic axis (PA $25^{\circ}$ ); see Fig. 7. The pv-diagram along the major axis shows evidence for solid body rotation for $r<2$ " (130 pc). The linear "pv" distribution along the major kinematic axis indicates that gas along this line is rotating with a single angular velocity. The turn-over of the velocity at larger radii $\left( \pm 4^{\prime \prime}\right)$ is not smooth, which implies that there might be some non-circular motions, or the presence of both $x_{1}$ and $x_{2}$ orbit families, belonging to a barred potential (e.g. Athanassoula \& Beaton 2006) present at this radius. More evidence for non-circular motions are found in the pv-diagram along the minor axis. If the gas was rotating with only circular velocities, then all velocities along the minor axis should be zero. This is not the case, in the $r<2^{\prime \prime}(130 \mathrm{pc})$ range there is some offset (up to $-50 \mathrm{~km} \mathrm{~s}^{-1}$ ). Also at larger radii deviations, up to $30 \mathrm{~km} \mathrm{~s}^{-1}$, along this axis are present, particular at $-5^{\prime \prime}$ to $-7^{\prime \prime}$ (that is, south of the nucleus). In both positionvelocity diagrams, some low-level emission, away from the main emission structure, is visible. Due to the low declination of the source, and the orientation of the major axis, the beam side lobes were oriented in the same direction as the emission in individual channels. This made it very difficult to completely remove them. Here we see the remainder, which is only at the $2 \sigma$ level.

\subsubsection{Non-circular gas motions}

We investigate the location of the non-circular motions in the gas in more detail. We use the GIPSY routine ROTCUR (van der Hulst et al. 1992; Vogelaar \& Terlouw 2001) to construct a rotation curve spanning the entire galaxy out to a radius of $24 \mathrm{kpc}$. We combine our $\mathrm{CO}(2-1)$ data with the BIMA SONG CO(1-0) and VLA HI data, so that this rotation curve has, at the same time, high resolution in the central kiloparsec.

A galaxies' observed line-of-sight velocity field can be decomposed into harmonic components, $V_{\text {los }}=V_{\text {sys }}+V_{\text {circ }}+V_{\text {rad }}+$ higher order terms (Canzian 1993; Schoenmakers et al. 1997), where $V_{\text {sys }}$ is the systemic velocity, $V_{\text {circ }}$ the pure rotational component to the velocity, and $V_{\text {rad }}$ the pure radial component to the velocity. The ROTCUR determines these velocity 

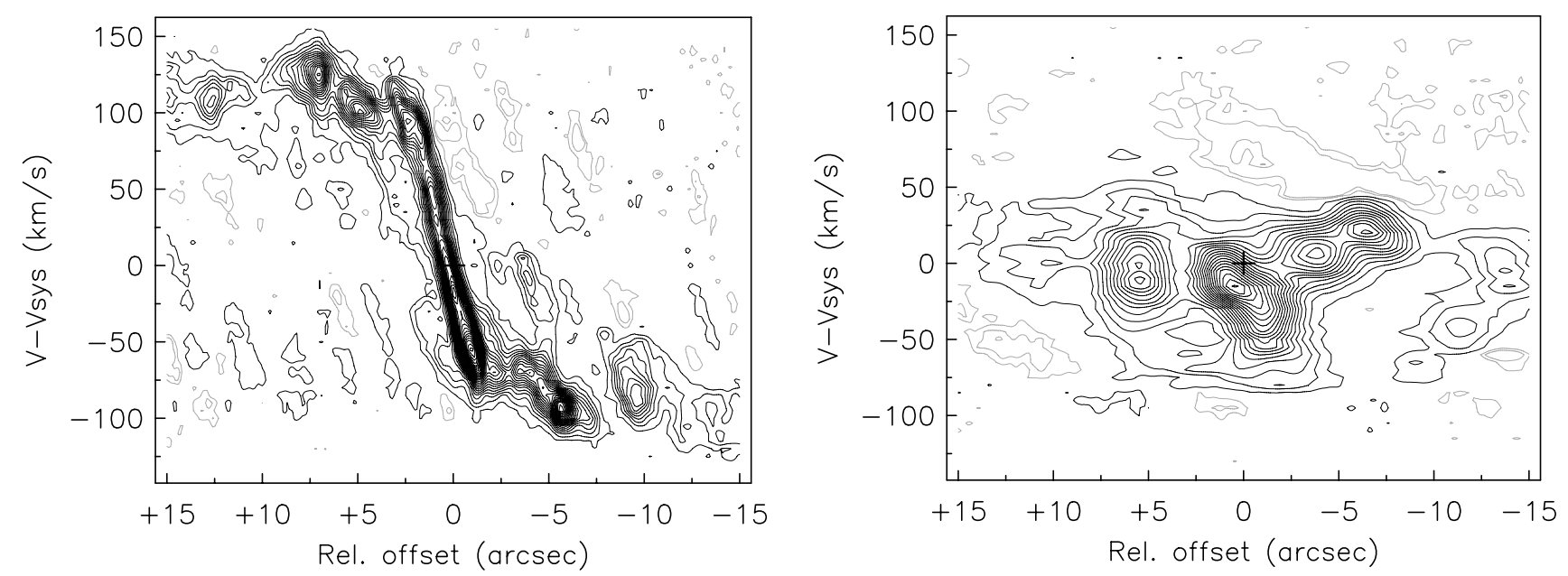

Fig. 7. Left: position-velocity diagram along the major axis $\left(\mathrm{PA}\right.$ of $115^{\circ}$ ) through the dynamic center. The cut was made from the SE (positive offsets) to the NW (negative offsets). Right: position-velocity diagram along the minor axis (PA of $25^{\circ}$ ) through the dynamic center, from the NE (positive offsets) to the SW (negative offsets). In both panels, the contours are at $2 \sigma$, in $2 \sigma$ steps. The dynamic center is at $(0,0)$.
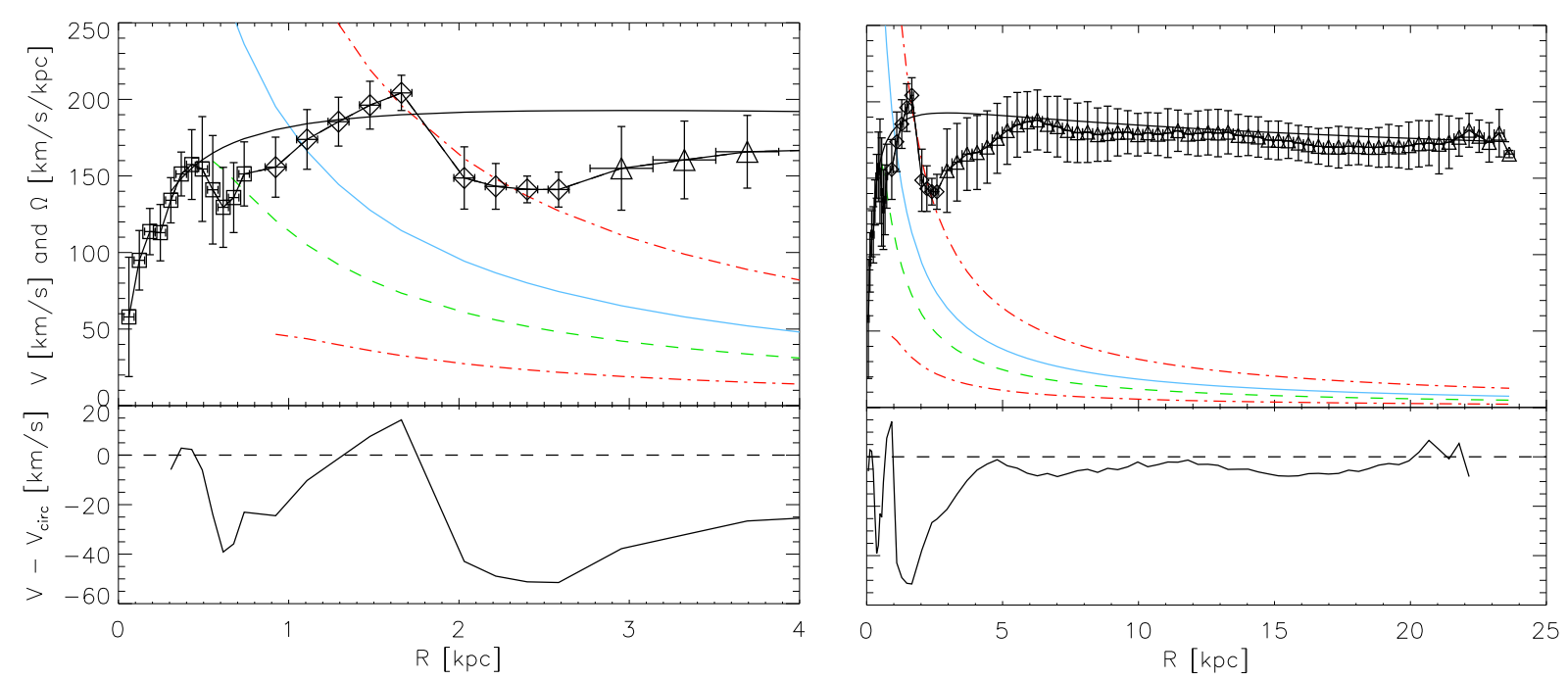

Fig. 8. Gas rotation curve of NGC 5248, spanning the inner $4 \mathrm{kpc}$ (left) and the entire disk out to $24 \mathrm{kpc}$ (right). The rotation curve is based on our $\mathrm{CO}(2-1)$ (squares), as well as BIMA SONG CO(1-0) (diamonds) and VLA HI (triangles) data, giving both high spatial resolution and large radial coverage. A circular velocity curve is fit using Eq. (1), solid line. Frequency curves of $\Omega$ (solid, blue), $\Omega+\kappa / 2, \Omega-\kappa / 2$ (both dash-dot, red) and $\Omega-\kappa / 4$ (dashed, green) are also presented. The bottom panels show the difference between circular velocity model curve and ROTCUR derived rotation curve. (See text for further details.)

components, $V_{\text {sys }}, V_{\text {circ }}$, and $V_{\text {rad }}$, by fitting tilted rings (parameterized with inclination, PA, and dynamical center) as a function of radius to the observed line-of-sight velocity field. (Higher order terms are not present in ROTCUR.) The systemic velocity $V_{\text {sys }}\left(1153 \mathrm{~km} \mathrm{~s}^{-1}\right)$ as well as the dynamical center and inclination $\left(43.1^{\circ}\right)$ of NGC 5248's disk were determined, and then fixed, in an initial run of ROTCUR on the HI data alone, since the HI observations have the larger extent and are therefore less sensitive to the expected radial motions in the central kiloparsec. Further investigation then showed no significant change in the PAs of the tilted rings fitted to the different gas tracers as function of radius, the values were in accordance with the major axis orientation, thus the PA was fixed to $115^{\circ}$ (the major axis orientation). Finally, we also kept $V_{\text {rad }}$ at zero, and let ROTCUR fit the only remaining variable, $V_{\text {circ }}$.

From the center to the edge of the PdBI FoV, the $\mathrm{CO}(2-1)$ velocity was sampled around the major axis every $1^{\prime \prime}$ out to $11^{\prime \prime}(620 \mathrm{pc})$, excluding a $20^{\circ}$ wedge around the minor axis. Similarly, the BIMA SONG velocity was sampled every $3^{\prime \prime}$ out to $42^{\prime \prime}(2.6 \mathrm{kpc})$. Finally, the HI velocity field was sampled every $6^{\prime \prime}$ out to $6.5^{\prime}(24 \mathrm{kpc})$. The derived velocities match very well at the radii where the different datasets overlap. Figure 8 shows both a zoom of the inner $4 \mathrm{kpc}$ (left) and the full rotation curve (right).

The resulting velocity curve now under-predicts the "true" circular velocity at those radii were radial motion is present, since we can reasonably assume that gas is moving inwards under the gravitational torques exerted by the stellar bar and/or spiral arms (i.e. $V_{\text {rad }}$ is expected to be less or equal to $0 \mathrm{~km} \mathrm{~s}^{-1}$ ). Therefore, a circular velocity curve of the following form (e.g. Faber \& Gallagher 1979) is fitted as an upper envelope to the peaks in the rotation curve (solid line in Fig. 8).

$V_{\text {circ }}(R)=\frac{V_{\max } \times\left(R / R_{\max }\right)}{\left[1 / 3+2 / 3 \times\left(R / R_{\max }\right)^{n}\right]^{3 n / 2}}$ 
with $V_{\max }=156 \mathrm{~km} \mathrm{~s}^{-1}, R_{\max }=440 \mathrm{pc}$ and $n=0.855$. This smooth profile is indicative of the real galactic rotation profile, if all material was only rotating on circular orbits. This functional form further gives smooth curves for $\Omega, \Omega-\kappa / 2$, etc. The difference between the fitted velocity and the model circular velocity is shown in the bottom panel of Fig. 8. At radii larger than $6 \mathrm{kpc}$ there is very little deviation from the circular velocity profile.

Inside $6 \mathrm{kpc}$ the offset reaches $-50 \mathrm{~km} \mathrm{~s}^{-1}$. In the inner $6 \mathrm{kpc}$ the fitted and model velocity curves overlap at two radii, $1.3-1.7 \mathrm{kpc}$ and $400 \mathrm{pc}$. To avoid resolution effects, we do not compute the difference within a conservative limit of $300 \mathrm{pc}$. The smaller radius can be immediately linked to the radius of the outer circumnuclear ring. At $\sim 1.5 \mathrm{kpc}$ there is no ring structure, but Haan et al. (2009) made a velocity decomposition of the HI velocity field and found a strong variation in the $s 3 / s 1$ term, which is indicative of a co-rotation (CR) radius (Canzian \& Allen 1997). Also, the BIMA SONG data showed that CO(1-0) emission was constrained to this radius and in the dust spiral shown in Fig. 4a of Jogee et al. (2002b) it does seem that the spiral's pitch angle changes significantly at this radius, indicating a change in gas/dust dynamics.

The $\Omega, \Omega+\kappa / 2, \Omega-\kappa / 2$ and $\Omega-\kappa / 4$ frequency curves, based on the fitted circular velocity curve, are also included in Fig. 8. The exact shape of the frequency curves depends on the derivative of the velocity curve. It is thus very sensitive to small variations. For this reason the curves within the central kiloparsec are not drawn. Further, Maciejewski (2004a) shows how the inclusion of a BH changes the frequency curves without changing the velocity curve. The $\Omega-\kappa / 2$ curve may show either a downturn followed by an upturn (constant density core), or an immediate upturn (MBH or density cusp). Circumnuclear star forming rings generally form near the ILR (e.g. Athanassoula 1992a; Regan \& Teuben 2003; Mazzuca et al. 2008; Kim et al. 2012), where this $\Omega-\kappa / 2$ curve intersects with the pattern speed of the asymmetric pattern that drives the gas inward. Jogee et al. (2002b) estimate that the pattern speed for their $5.9 \mathrm{kpc}$ large scale stellar bar must be close to $30 \mathrm{~km} \mathrm{~s}^{-1} \mathrm{kpc}$ given the semi-major axis of that bar; a bar's CR radius is typically at $1.2 \pm 0.2$ times the semi-major axis of the bar (Athanassoula 1992a). If the pattern speed is $30 \mathrm{~km} \mathrm{~s}^{-1} \mathrm{kpc}$, then the bar's CR would be at $7 \mathrm{kpc}$ and the (outer/only) ILR would be at $1.6 \mathrm{kpc}$.

Yuan \& Yang (2006) have also derived frequency curves for NGC 5248 by application of their nonlinear asymptotic theory of spiral density waves. Their results are based on $\mathrm{H} \alpha$ observations of the center, the Jogee $\mathrm{CO}(1-0)$ data, and the BIMA SONG CO(1-0) observations and extend out to $6 \mathrm{kpc}$. The frequency curves derived in this work lead to two ILR, with the oILR at $3 \mathrm{kpc}$ and the iILR very close to the nucleus. However, the same caveats as above should be taken into account here.

As a final investigation of the gas kinematic, we obtained the $2 \mathrm{D}$ distribution of non-circular motions in the $\mathrm{CO}(2-1)$ velocity field (Fig. 9). The residual velocity field is obtained by subtracting the fitted circular velocity, despite the caveats, from the observed velocity distribution. The residual field is dominated by the spiral arm structure (in red) in the south. This southern spiral arm shows residual velocities of up to $40 \mathrm{~km} \mathrm{~s}^{-1} / \sin (i)=60 \mathrm{~km} \mathrm{~s}^{-1}$. The northern spiral arm also shows non-circular motions (blue), but to a smaller extent, implying that the inflow is not symmetric along the large scale bar major axis. Similar asymmetries were present along the one-sided non-zero velocities in the minor axis pv-diagram (Fig. 7). In the west, connecting the outer and inner rings, the gas there also shows non-circular motions. These non-circular motions indicate that there is gas flow from the outer to the

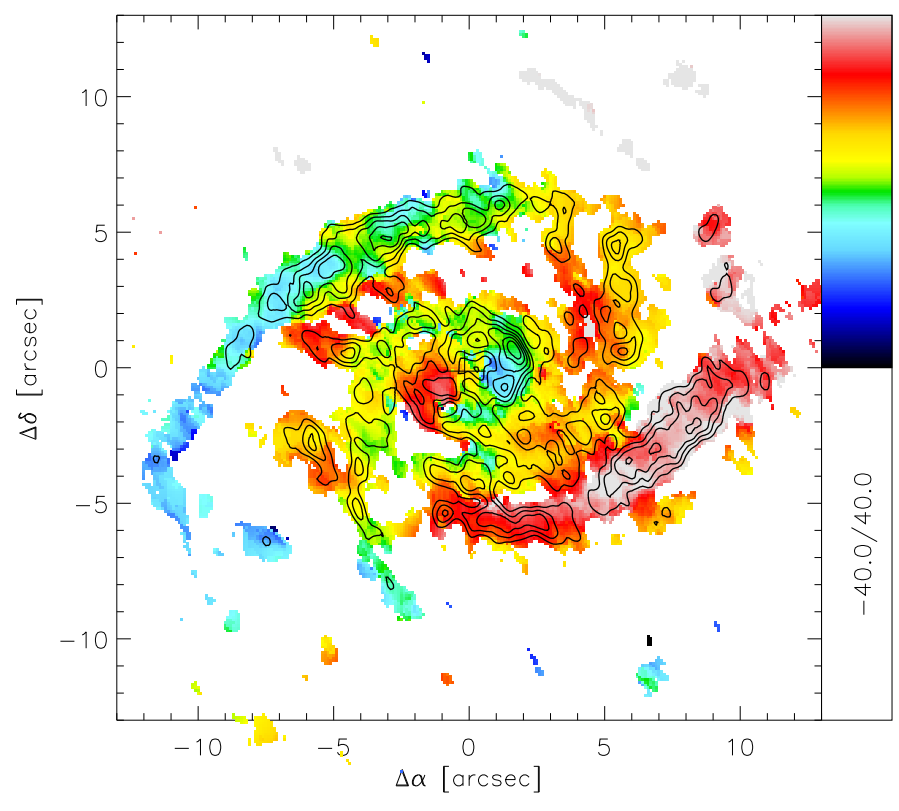

Fig. 9. $\mathrm{CO}(2-1)$ residual velocity field, obtained by subtracting the modeled circular velocity from the observed $\mathrm{CO}(2-1)$ velocity field. Overlaid are the flux contours of the integrated $\mathrm{CO}(2-1)$ emission. Contours start at $5 \sigma$, the residual velocity field was calculated at each position where the observed velocity field exceeded a $2 \sigma$ measurement. A color version of this figure is available in the electronic version.

inner circumnuclear ring. Comparison with the velocity dispersion field (Fig. 6) shows no corresponding increase in velocity dispersions. It is not clear if this non-circular motion is connected to the northern streaming spiral arm. Finally, at the inner ring radius, we reach the resolution limit and are hesitant to overinterpret the bipolar distribution seen with positive residuals in the east and negative ones in the west, as implying outflow.

\section{Stars and star formation in the center of NGC 5248}

In the previous section we revealed a complex pattern of radial motions that shows that gas flows inward from the outer to the inner circumnuclear ring. In this section, we derive the ages of the stellar populations in and around both rings, as well as their star formation histories, in order to further investigate the ring properties.

\subsection{Ages of the stellar clusters in the rings}

Maoz et al. (2001) detected 507 star cluster candidates in and around the two circumnuclear star forming ring in NGC 5248. They extracted their fluxes via aperture photometry in the following broadband filters: F220W, F336W, F547M, F814W and $F 160 W$. Here, we use a $\chi^{2}$ fitting routine and Starburst 99 models also used in Van der Laan et al. (2013) to re-determine the ages, masses and color excess for each star cluster in that set. The parameters of the Starburst 99 models are: Starburst 99 version v6.0.2 (Leitherer et al. 1999; Vázquez \& Leitherer 2005; Leitherer et al. 2010), run in burst mode, with a Kroupa IMF, solar metallicity, and Padova AGB solar metallicity tracks. Output was generated every $2 \mathrm{Myr}$ years. The new results that are presented in this work mainly reflect the improvements in stellar models over the past 10 years since the results of Maoz et al. (2001) were published. We also included nebular emission in 
Table 3. Star cluster ages, extinctions and masses in the circumnuclear star forming rings of NGC 5248.

\begin{tabular}{lcccc}
\hline \hline ID & Age [Myr] & $E(B-V)$ & $\log \left(\mathrm{Mass} / M_{\odot}\right)$ & $\chi^{2} /$ d.o.f. \\
\hline 001 & 6 & 0.00 & 4.8 & 18.29 \\
002 & 90 & 0.05 & 5.4 & 12.98 \\
003 & 16 & 0.00 & 4.8 & 10.23 \\
004 & 38 & 0.06 & 5.0 & 4.19 \\
005 & 22 & 0.38 & 5.0 & 10.87 \\
006 & 46 & 0.12 & 5.0 & 3.13 \\
007 & 6 & 0.00 & 4.0 & 6.31 \\
008 & 6 & 0.15 & 4.0 & 5.60 \\
009 & 6 & 0.20 & 4.0 & 6.93 \\
010 & 52 & 0.00 & 4.8 & 10.87 \\
011 & 28 & 0.00 & 4.6 & 7.61 \\
012 & 50 & 0.04 & 4.8 & 9.20 \\
013 & 6 & 0.05 & 3.8 & 6.54 \\
014 & 6 & 0.25 & 4.0 & 3.87 \\
015 & 42 & 0.00 & 4.6 & 4.16 \\
016 & 34 & 0.07 & 4.6 & 0.34 \\
017 & 44 & 0.00 & 4.6 & 5.41 \\
018 & 22 & 0.37 & 4.6 & 12.36 \\
019 & 26 & 0.00 & 4.4 & 3.31 \\
020 & 122 & 0.18 & 5.0 & 4.50 \\
\hline
\end{tabular}

Notes. Results of the $\chi^{2}$ fitting of age (Col. 2), color excess (Col. 3), and mass (Col. 4) of the first 20 observed star clusters of NGC 5248. The star cluster ID in Col. 1 is equal to that in the Maoz et al. (2001) electronic table. The full table is available at the CDS.

the model fluxes, which adds significant emission for young ages at, predominantly, the shorter wavelengths. Our photometric library also contains a wider age range than the original $\mathrm{Maoz}$ et al. (2001) work. The generated model spectra were weighted with a range of $E(B-V)$ values $([0,3]$ in 0.01 steps) and stellar masses $\left(\left[10^{3}-10^{7} M_{\odot}\right]\right.$, in 0.2 dex steps). Table 3 gives the fitted ages (Col. 2), color excesses (Col. 3) and masses (Col. 4) for the first 20 star clusters (the full table is available at the CDS) that contained detections in 3 or more bands and were successfully fitted. Column 1 contains the original ID of each star cluster. In total 308 candidates were successfully fitted, $\chi^{2}$ given in Col. 5 .

In comparison with previous results from Maoz et al. (2001), we find that the star clusters

- have slightly lower $\chi^{2}$ values;

- are on average $30 \%$ heavier;

- are older (>50 Myr); and

- have similar color excess.

There are several factors that can explain why we obtain heavier and older star clusters. Our analysis uses a Kroupa IMF, while Maoz et al. (2001) use a Salpeter IMF. A Kroupa IMF has a higher fraction of low-mass stars. Most of the luminosity is contained in the high mass stars, so low mass stars can increase the total mass of a cluster without (significantly) increasing the luminosity. This result holds even when we take the difference in high mass cut-offs in the chosen IMFs into account $\left(120 \mathrm{M}_{\odot}\right.$ by Maoz, $100 M_{\odot}$ by us). A second factor that changes the derived star cluster mass is that our fitting routine does not find as many young star cluster ages. The inclusion of nebular emission boosts the fluxes at the short wavelength side of the younger model SEDs and fewer star clusters fit that distribution. As a direct result, the fitted mass of the star cluster must increase, since the mass-to-light ratio increases with age. These two factors in mass "gain" are offset by a difference in the adopted distance to the galaxy. In this work a newer distance measurement of 12.7 Mpc for NGC 5248 (Tully et al. 2009) is adopted, while Maoz et al. (2001) adopted a distance of $22.7 \mathrm{Mpc}$. The shorter distance would lower our derived cluster masses by about a factor of 3. Combined, these factors culminate in the $30 \%$ more massive and somewhat older star clusters found in the current fitting.

In Fig. 10 (left panel) the star cluster fitting output is visualized for age. The position of each star cluster is relative to the dynamic center and the positions have been deprojected. The derived ages are split into six age ranges, the color hue of each bin decreases with age. The youngest $(<10 \mathrm{Myr})$ star clusters are found in the larger ring, in its west and east quadrants. Older star clusters are distributed uniformly throughout the larger ring. At the inner ring, only seven star clusters were successfully fitted. There, the ages of the clusters start in the 10-20 Myr age range and increase along a clockwise gradient from the north. The derived masses of the star clusters are shown in the middle panel. The results are binned by $0.5 \mathrm{dex}$. The star clusters have an average stellar mass of $10^{4.5} M_{\odot}$. No significant mass variation as a function of azimuth can be seen in either ring. Finally, the derived color excesses are plotted in Fig. 10 (right panel). The color hue increases with increasing color excess. The color excess is mostly uniformly distributed throughout the rings. Star clusters with lower color excess are primarily found in the north and at the outer edge of the larger ring. At the inner ring again a clockwise gradient is seen, with increasing color excess from the north.

A comparison of the age and color excess azimuthal gradient in the smaller ring with the strongest emission of the $\mathrm{CO}(2-1)$ line shows that the highest extinction is partly cospatial with the $\mathrm{CO}(2-1)$ emitting gas. The youngest star clusters in this inner circumnuclear ring are positioned at a slightly larger clock-wise angle. Therefore, star formation appears predominantly to occur at one location. No [FeII] emission was detected from the inner ring (Böker et al. 2008). [FeII] emission is believed to trace supernova activity, which starts $\geq 10 \mathrm{Myr}$ after star formation. The lack of [FeII] emission, combined with the age gradient of star clusters found in this ring, suggests that star formation is intermittent, possibly periodic, in the inner ring. In Sect. 5.2 we speculate how this gradient of extinction, gas and young stars could be generated.

\subsection{Stellar populations from SAURON observations}

The star cluster analysis above is supplemented with an analysis of the underlying stellar population as traced by SAURON IFU observations. The flux at each SAURON spaxel contains the contributions of all stellar populations at those positions, including stars that are not in any of the detected star clusters. The SAURON data cube was originally fitted with a full range of age and metallicity SSP models from the MILES database ${ }^{5}$. The resulting maps are shown in Fig. 4. This large fit shows that solar metallicity is the predominant metallicity in the central region of this galaxy. A fitting residual of $\sim 1.5 \%$ of the flux remains in each spaxel when fitting with this full range of SSP models.

Following Van der Laan et al. (2013), a smaller combination of SSP spectra, at solar metallicity, is fitted to the SAURON data cube. The goal is to separate the stellar light at each spaxel into "young", "intermediate" and "old" components, and determine if the rings stand out in recent star formation. For this reason, four SSP model spectra are selected. The four SSPs chosen have

http://miles.iac.es 

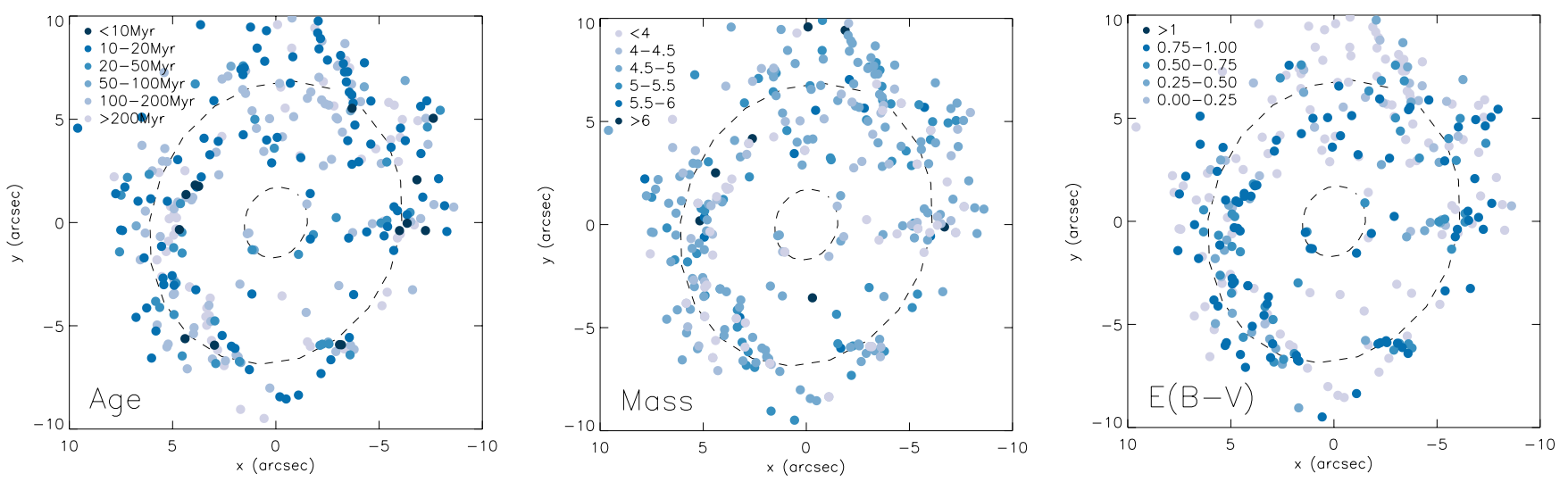

Fig. 10. Results of the $\chi^{2}$ fitting. Left derived ages, middle derived masses, right derived color excess. The positions of the star clusters are relative to the dynamic center and have been deprojected.

solar metallicity and have designated model ages of $70.8 \mathrm{Myr}$, 158.5 Myr, 398.1 Myr and 3.2 Gyr. Van der Laan et al. determined that these four SSP spectra reasonably span the full possible stellar age range. The emission lines, $\mathrm{H} \beta$, [OIII], and [NI] are also included in this alternative fitting to avoid contamination of the SSP results by gaseous emission.

The output from the fitting routines pPXF (Cappellari \& Emsellem 2004; Emsellem et al. 2004) and GANDALF (Sarzi et al. 2006) contains the number weighted fractions of each SSP spectrum at each spaxel. From these number weighted fractions the mass weighted fractions at each position are determined, via the luminosity of each SSP spectrum and its massto-light ratio. This mass fraction is multiplied with the stellar intensity at each position to give an "absolute" mass distribution in each SSP age bin. The age bins are designated "young", "intermediate I", "intermediate II" and "old". We iterate the fitting 30 times. Random noise on the level of the initial fitting residual ( $1.5 \%$ of the flux) is introduced at each iteration. In this manner, a mass fraction uncertainty of less than $5 \%$ can be reached. The results are presented in Fig. 11.

In the "young" age bin, high stellar masses are predominantly seen at two locations opposite each other at the radius of the outer ring. The majority of the young star clusters are also found at these locations. At the inner ring some lower fractions of young stars are seen.

The "intermediate" age bins show the least stellar mass of all panels. Van der Laan et al. (2013) investigated this. The fitting routine minimizes the number of SSP spectra needed to achieve an acceptable fit. Therefore, the high(est) contrast between "young" and "old" is used when possible. Nevertheless, some intermediate age stellar populations are found at both the outer and inner circumnuclear ring. The "intermediate II" bin shows a significant fraction of stellar mass in the NE quadrant of the outer ring. This is between the two locations of high "young" stellar mass and co-spatial with one of the high $1 \mathrm{~mm}$ emission peaks as seen in Fig. 2. Neither ring is visible as such in the "old" stellar mass map. The "old" distribution shows a "bulgelike" profile, peaking in the center.

\section{Discussion}

In understanding the make-up of the central region in NGC 5248 we need to account for both the gaseous and stellar distribution, as well as the star formation that connects then. To this end we first discuss the locations were star formation occurs.
Afterwards, we discuss which components are a likely part of the central region of this galaxy.

\subsection{Star formation scenarios in the circumnuclear rings}

When gas is driven onto a circumnuclear ring due to driving by a stellar bar, two star formation scenarios in circumnuclear rings are proposed. They are called "popcorn" and "pearls-on-astring". The difference between the two lies in the assumed time between gas inflow and the onset of star formation. The "popcorn" scenario was proposed by Elmegreen (1994) and holds that the gas density in the ring builds up throughout the ring to some critical density, at which point star formation starts uniformly throughout the ring. The second scenario was put forward by Böker et al. (2008) and assumes that star formation will occur predominantly close to the two "inflow" or overdensity points of gas onto the ring. The argument is that the gas density will be higher where the gas spiral arms and ring connect than elsewhere in the ring and thus that star formation will mainly occur there. As the new stars are moving slowly out of the overdensity regions, a string of aging stellar populations would be formed in the ring. Evidence for the "pearls-on-a-string" scenario in one or both rings is thus an indication for the origin of that ring to be due to gravitational torques from a stellar bar.

In both the star cluster analysis and the SAURON fitting, the youngest stellar populations are found at two locations. These positions are north-west and south-east at the radius of the larger ring. They are anti-correlated with the $\mathrm{CO}$ emission at this radius, which could imply that molecular gas is almost fully converted into stars as it enters the ring, the "pearls-on-a-string" scenario. Arguments for "pearls" may further be found in the $1 \mathrm{~mm}$ continuum map (Fig. 2). Two high emission regions are present in the $1 \mathrm{~mm}$ continuum distribution at the radius of the larger circumnuclear star forming ring. $1 \mathrm{~mm}$ emission arises most likely from cold dust and traces the bulk of the gas mass in the circumnuclear region. The combination of the high emission regions in the $1 \mathrm{~mm}$ continuum map/CO spiral arms and the young stellar mass concentration would form two "pearls" of the "pearls-ona-string" scenario; current star formation and recent star formation. The contact points of the spiral arms generated by the Jogee et al. $5.9 \mathrm{kpc}$ large scale stellar bar $\left(\mathrm{PA}=135^{\circ}\right)$ should have gas entering the larger circumnuclear ring at the north and south. This corresponds with our observations only if star formation is somewhat delayed (a quarter rotation, 5 Myr). 

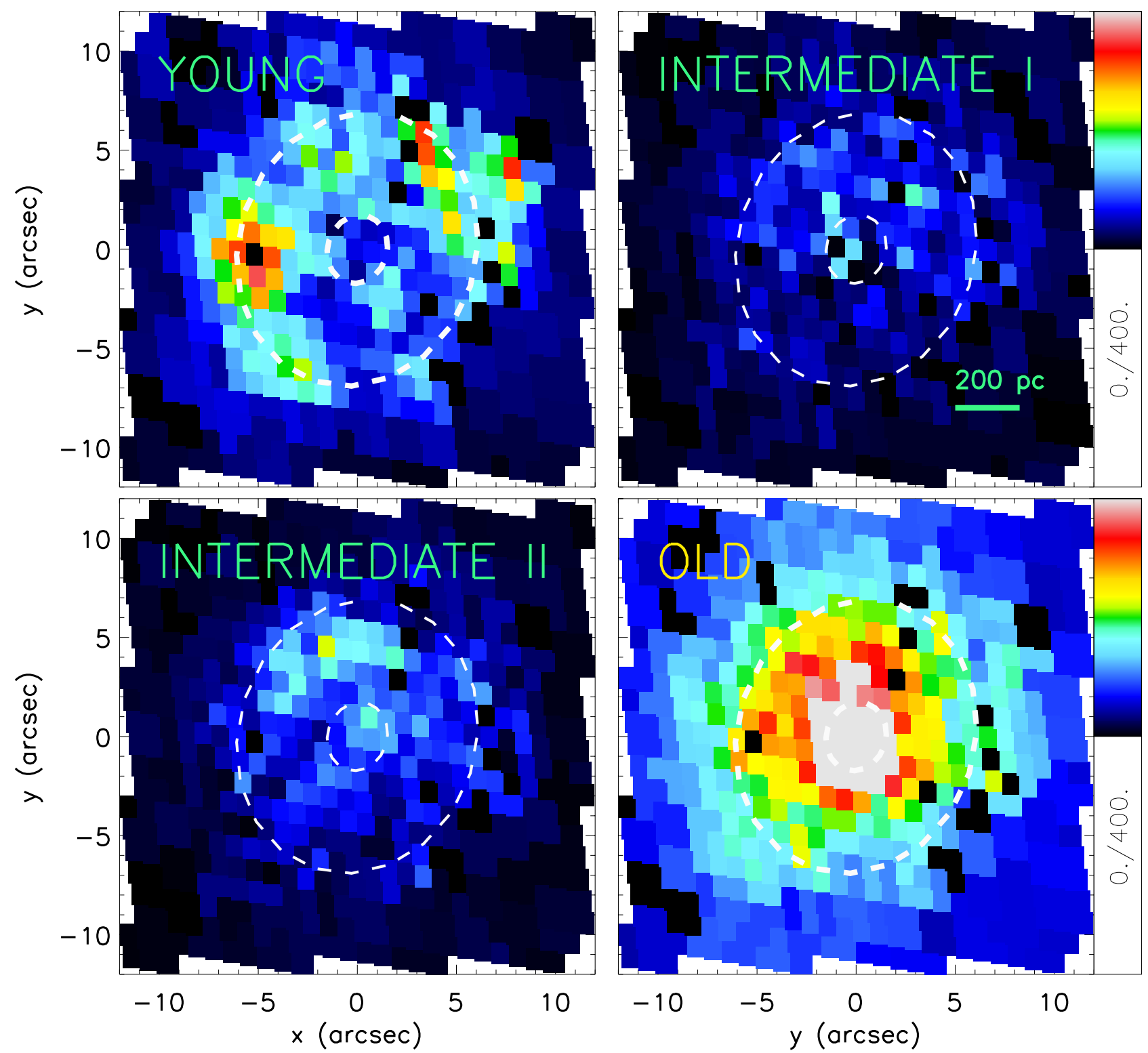

Fig. 11. Stellar "mass" maps in [A.U.] for the 4 age bins fitted to the SAURON IFU data set. The maps have been corrected for inclination and the positions of the two circumnuclear rings (1.5" and 6") have been indicated. A color version of this figure is available in the electronic version.

There are some indications that the star formation in the inner ring also shows sites of preferred star formation. The fitted star clusters are found exclusively in the western-southern half of the ring. A sequence was discussed in Sect. 4.1, going from position with the highest extinction (which could be interpreted as an overdensity point), followed by/partly co-spatial with the $\mathrm{CO}(2-1)$ emitting gas, followed by the location of the youngest star clusters in this inner circumnuclear ring. In analogy with the outer circumnuclear ring, there would be 3 "pearls", but starting only at one contact point!

It is not possible to obtain a more quantitative observation of the "pearls-on-a-string" scenario. Orbital timescales in the central kiloparsec are $\frac{\pi}{v\left[\mathrm{~km} \mathrm{~s}^{-1}\right] / r[\mathrm{kpc}]} \sim 5-20 \mathrm{Myr}$. At the same time, a reasonable timescale for a large scale bar pattern to complete one rotation, which sets the rotation of the overdensity points, is $\sim 100$ Myr. In one rotation of the pattern, the newly formed stars would have made several full rotations already. This makes detecting any stellar age gradient beyond the very crude and broad ones just discussed, impossible. However, we should also not overweight the "pearl" observation, especially at the inner ring. Since only one contact point is found, and the dynamical times are truly very short, it is still possible that this is a transient configuration.

\subsection{What components drive the central region?}

Gravitational mechanisms dominate gas transport on kiloparsec scales (García-Burillo et al. 2005; Haan et al. 2009), so our first focus is on identifying the dominant mass component. The gravitational potential in the central $1.5 \mathrm{kpc}$ in NGC 5248 is dominated by the stellar mass, as can be concluded from the following simple calculation. The dynamical mass $M_{\text {dyn }}$ is $\sim 8.5 \times 10^{9} M_{\odot}$ at $1.5 \mathrm{kpc}$ radius, of which the atomic $(\mathrm{HI})$ and molecular $(\mathrm{CO})$ gas together make up $10 \%$. This implies that gas self-gravity can be largely ignored and that the (molecular) gas distribution is shaped by the stellar gravitational potential.

Jogee et al. (2002b) established the current understanding of this system with their discovery of a $95^{\prime \prime}(5.9 \mathrm{kpc})$ stellar bar. 
This "weak" (ellipticity 0.44) oval/bar drives two spiral arms inside its CR. These arms show star formation between $30^{\prime \prime}$ and $90^{\prime \prime}$. Since star formation requires that gas is not "streaming", it is unlikely that there is significant radial gas flow inwards at these radii. However, this spiral arm/bar system also is visible in dust maps at radii between $6^{\prime \prime}$ and $70^{\prime \prime}$. Between $66^{\prime \prime}$ and $30^{\prime \prime}$ there is no star formation in these spiral arms. Jogee et al. (2002b) argue that the large-scale stellar bar drives an Englmaier \& Shlosman (2000)-type density wave spiral that crosses the ILR of the bar pattern at $26^{\prime \prime}(1.6 \mathrm{kpc})$ and accounts for the full distribution of the molecular gas in this system.

In the case of this scenario, we have an objection to the extent of the nuclear spiral. Jogee et al. (2002b) extend the density wave spiral down to the smaller circumnuclear ring at $1.5^{\prime \prime}$ (100 pc). This argument is based on two observations. Laine et al. (1999) detected a grand-design nuclear spiral in NIR $J$-, $H$-, and $\mathrm{K}$-band observations. However, the luminosity contrast between arm and inter-arm regions is only $0.05 \mathrm{mag}$. We therefore hold it to be tentative. The second indicator is the existence of a "patchy" spiral arm seen in a $K_{\mathrm{s}}$-band (Jogee et al. 2002b) observation of the region between the two circumnuclear rings, that could be connected to one of the spiral arms at larger radii. The presence of a second arm, that connects to the other spiral, is not clear from those observations. Yuan \& Yang (2006) also determined the form of the spiral density wave and claimed its existence to $100 \mathrm{pc}$. Those authors acknowledge that, possibly due to loss of resolution, the spiral merges into an oval feature at these radii.

We do find non-circular motion between the two rings in our $\mathrm{CO}(2-1)$ observations. Indeed, such motion is necessary to fuel the current star formation at the smaller circumnuclear ring. However, we find no clear indication in our data that the molecular gas is indeed still driven by the density spiral wave inside the $5^{\prime \prime}(370 \mathrm{pc})$ star forming ring. Specifically, because it is a star forming ring with clear indications that gas is rapidly being converted into stars, the "pearls-on-a-string" scenario discussed in the previous section.

An alternative scenario may be the following. A dynamical analysis by Haan et al. (2009) found 2 CR in NGC 5248, at $28-30^{\prime \prime}(1.7-2.0 \mathrm{kpc})$ and $120-185^{\prime \prime}(7.5-12 \mathrm{kpc})$, indicating two patterns. The latter may indeed refer to the large scale stellar bar/spiral arms, but the first is still to be explained. The 28-30" radius is similar to the star formation transition, as well as the BIMA SONG $\mathrm{CO}(1-0)$ molecular gas limit of $22^{\prime \prime}(1.4 \mathrm{kpc})$.

Earlier estimates (e.g. Martin 1995) of a stellar bar in NGC 5248 were of a smaller bar, only $22^{\prime \prime}(1.4 \mathrm{kpc})$ in length, but with similar PA to the $95^{\prime \prime}(5.9 \mathrm{kpc})$ bar. There are several arguments to reconsider the inclusion of this smaller bar into our understanding of this system. It explains the second CR. Also, the transition at $30^{\prime \prime}$ from non star forming to star forming spiral arms is explained. Inside the CR of this smaller bar, the spiral arms would indeed be dominated by shocks, thus nonstar forming and given to streaming motion. Further, it is much more probably that the $6^{\prime \prime}(370 \mathrm{pc})$ circumnuclear ring is near the (i)ILR of this smaller stellar bar.

It is our belief that the formation of the smaller circumnuclear ring at $100 \mathrm{pc}$ remains undetermined. Given the strong star formation at the larger ring, it is very possible that the increased radiation pressure and/or turbulence of the young stars leads to gas overcoming the barrier and moving further in. These hydrodynamical forces especially play a role when a significant radial density gradient is present, as in a circumnuclear ring. The bimodal structure of the star formation in the larger ring could also explain the Laine et al. (1999) grand-design nuclear spiral observation. Finally, due to the presence of an SMBH at the center, a nuclear resonance would halt the inflowing gas again before reaching the nucleus proper.

Either scenario would be consistent with the observed stellar and gaseous distribution in the central region of this galaxy.

\section{Summary}

The central region of the barred spiral galaxy NGC 5248 contains two circumnuclear star forming rings at $1.5^{\prime \prime}(100 \mathrm{pc})$ and $6 "$ (370 pc) from its quiescent nucleus. This distribution is surprising, since circumnuclear star forming rings are considered to be efficient gas barriers. We combine analyses of the gaseous and stellar content in the central kiloparsec of this galaxy to understand the gas distribution and dynamics of this star forming central region. We present new $\mathrm{PdBI}+30 \mathrm{~m} \mathrm{CO}(2-1)$ emission line observations that show two spiral arms at the larger ring radius, and a gas ring at the smaller ring radius. This is the first time that the $100 \mathrm{pc}$ circumnuclear ring is resolved in molecular gas observations.

From the stellar analysis it has become clear that the 6 " $(370 \mathrm{pc})$ star forming ring is a "true" circumnuclear ring. We find evidence of the "pearls-on-a-string" star formation scenario, which implies that gas driven onto this ring is quickly converted into stars. Further, we find that there is radial gas motion between the two rings, consistent with current star formation at the inner ring.

The current understanding of this region comes from Jogee et al. (2002b). They propose that the gas distribution is dominated by a spiral density wave driven by a large scale stellar bar of 95" (5.9 kpc). Following Englmaier \& Shlosman (2000) this density wave crosses the ILR at $26^{\prime \prime}(1.6 \mathrm{kpc})$ radius to become a nuclear spiral. This nuclear spiral is believed to continue down to $1.5^{\prime \prime}(100 \mathrm{pc})$ and thus fuel both star forming rings.

We propose an alternative scenario for consideration. Resurrection of a stellar bar previously believed to be present (Martin 1995), which has an extent to $22^{\prime \prime}$ (1.4 kpc), in combination with the larger stellar bar/spiral arms. This stellar bar would explain the two CR found by Haan et al. (2009), as well as provide an alternative explanation for the transition from not star forming to star forming at $30^{\prime \prime}(1.8 \mathrm{kpc})$ radius in the spiral arms. Furthermore, a smaller bar is better placed to explain the location of the $6^{\prime \prime}(370 \mathrm{pc})$ star forming ring.

For the origin of the $1.5^{\prime \prime}(100 \mathrm{pc})$ star forming ring, we believe there is not enough evidence to conclude a nuclear spiral wave. Viscous forces at the larger ring are equally probable to have brought gas further inward. The resonance near which this smaller ring forms is most likely a nuclear resonance with the SMBH.

Acknowledgements. We thank the referee for his/her comments that helped improve this paper. T.v.d.L. was in part supported by DFG-funding (grant SCHI 536/2-3). This work is based on observations carried out with the IRAM Plateau de Bure Interferometer. IRAM is supported by INSU/CNRS (France), MPG (Germany) and IGN (Spain). We would like to thank the staff at IRAM Spain, especially A. Sievers and M. Gonzalez, for invaluable help with the $30 \mathrm{~m}$ observations, and our local contact for the PdBI at IRAM France, J.M. Winters. We also thanks D. Maoz for making his HST observations available to us. This research has made use of the NASA/IPAC Extragalactic Database (NED) which is operated by the Jet Propulsion Laboratory, California Institute of Technology, under contract with the National Aeronautics and Space Administration.

\section{References}

Armour, J. N., \& Ballantyne, D. R. 2012, ApJ, 752, 87 Athanassoula, E. 1992a, MNRAS, 259, 328 
Athanassoula, E. 1992b, MNRAS, 259, 345

Athanassoula, E. 2000, in Stars, Gas and Dust in Galaxies: Exploring the Links, eds. D. Alloin, K. Olsen, \& G. Galaz, ASP Conf. Ser., 221, 243

Athanassoula, E., \& Beaton, R. L. 2006, MNRAS, 370, 1499

Bacon, R., Copin, Y., Monnet, G., et al. 2001, MNRAS, 326, 23

Bautista, M. A. 1999, ApJ, 527, 474

Berman, R. H., Pollard, D. J., \& Hockney, R. W. 1979, A\&A, 78, 133

Böker, T., Falcón-Barroso, J., Schinnerer, E., Knapen, J. H., \& Ryder, S. 2008, AJ, 135, 479

Boone, F., Baker, A. J., Schinnerer, E., et al. 2007, A\&A, 471, 113

Braine, J., Combes, F., Casoli, F., et al. 1993, A\&AS, 97, 887

Canzian, B. 1993, ApJ, 414, 487

Canzian, B., \& Allen, R. J. 1997, ApJ, 479, 723

Cappellari, M., \& Emsellem, E. 2004, PASP, 116, 138

Casasola, V., Combes, F., García-Burillo, S., et al. 2008, A\&A, 490, 61

Casasola, V., Hunt, L. K., Combes, F., et al. 2010, A\&A, 510, A52

Combes, F. 2008, in Formation and Evolution of Galaxy Disks, eds. J. G. Funes, \& E. M. Corsini, ASP Conf. Ser., 396, 325

Combes, F., García-Burillo, S., Boone, F., et al. 2004, A\&A, 414, 857

Combes, F., Baker, A. J., Schinnerer, E., et al. 2009, A\&A, 503, 73

de Vaucouleurs, G., de Vaucouleurs, A., Corwin, Jr., H. G., et al. 1991, Third

Reference Catalogue of Bright Galaxies (New york: Springer-verlag)

Dumas, G., Mundell, C. G., Emsellem, E., \& Nagar, N. M. 2007, MNRAS, 379, 1249

Elmegreen, B. G. 1994, ApJ, 425, L73

Emsellem, E., Cappellari, M., Peletier, R. F., et al. 2004, MNRAS, 352, 721

Englmaier, P., \& Shlosman, I. 2000, ApJ, 528, 677

Englmaier, P., \& Shlosman, I. 2004, ApJ, 617, L115

Erwin, P. 2004, A\&A, 415, 941

Erwin, P., \& Sparke, L. S. 2002, AJ, 124, 65

Faber, S. M., \& Gallagher, J. S. 1979, ARA\&A, 17, 135

Falcón-Barroso, J., Sánchez-Blázquez, P., Vazdekis, A., et al. 2011, A\&A, 532, A95

Ferland, G. J., Henney, W. J., O’Dell, C. R., et al. 2012, ApJ, 757, 79

García-Burillo, S., Combes, F., Eckart, A., et al. 2003a, in Active Galactic Nuclei: From Central Engine to Host Galaxy, eds. S. Collin, F. Combes, \& I. Shlosman, ASP Conf. Ser., 290, 423

García-Burillo, S., Combes, F., Hunt, L. K., et al. 2003b, A\&A, 407, 485

García-Burillo, S., Combes, F., Schinnerer, E., Boone, F., \& Hunt, L. K. 2005, A\&A, 441, 1011

García-Burillo, S., Fernández-García, S., Combes, F., et al. 2009, A\&A, 496, 85

Haan, S., Schinnerer, E., Mundell, C. G., García-Burillo, S., \& Combes, F. 2008, AJ, 135, 232

Haan, S., Schinnerer, E., Emsellem, E., et al. 2009, ApJ, 692, 1623

Helfer, T. T., Thornley, M. D., Regan, M. W., et al. 2003, ApJS, 145, 259

Hunt, L. K., Combes, F., García-Burillo, S., et al. 2008, A\&A, 482, 133
Huntley, J. M., Sanders, R. H., \& Roberts, Jr., W. W. 1978, ApJ, 221, 521

Jogee, S., Knapen, J. H., Laine, S., et al. 2002a, ApJ, 570, L55

Jogee, S., Shlosman, I., Laine, S., et al. 2002b, ApJ, 575, 156

Kim, W.-T., Seo, W.-Y., Stone, J. M., Yoon, D., \& Teuben, P. J. 2012, ApJ, 747, 60

Koda, J., Scoville, N., Hasegawa, T., et al. 2012, ApJ, 761, 41

Krips, M., Eckart, A., Neri, R., et al. 2005, A\&A, 442, 479

Laine, S., Knapen, J. H., Perez-Ramirez, D., Doyon, R., \& Nadeau, D. 1999, MNRAS, 302, L33

Laine, S., Knapen, J. H., Pérez-Ramírez, D., Englmaier, P., \& Matthias, M. 2001, MNRAS, 324, 891

Leitherer, C., Schaerer, D., Goldader, J. D., et al. 1999, ApJS, 123, 3

Leitherer, C., Ortiz Otálvaro, P. A., Bresolin, F., et al. 2010, ApJS, 189, 309

Lindt-Krieg, E., Eckart, A., Neri, R., et al. 2008, A\&A, 479, 377

Maciejewski, W. 2004a, MNRAS, 354, 883

Maciejewski, W. 2004b, MNRAS, 354, 892

Maciejewski, W., \& Small, E. E. 2010, ApJ, 719, 622

Maciejewski, W., Teuben, P. J., Sparke, L. S., \& Stone, J. M. 2002, MNRAS, 329,502

Maoz, D., Barth, A. J., Ho, L. C., Sternberg, A., \& Filippenko, A. V. 2001, AJ, 121,3048

Martin, P. 1995, AJ, 109, 2428

Mazzuca, L. M., Knapen, J. H., Veilleux, S., \& Regan, M. W. 2008, ApJS, 174, 337

Pogge, R. W., \& Martini, P. 2002, ApJ, 569, 624

Regan, M. W., \& Teuben, P. 2003, ApJ, 582, 723

Sánchez-Blázquez, P., Peletier, R. F., Jiménez-Vicente, J., et al. 2006, MNRAS, 371,703

Sandstrom, K. M., Leroy, A. K., Walter, F., et al. 2012, ApJ, submitted [arXiv: 1212.1208]

Sarzi, M., Falcón-Barroso, J., Davies, R. L., et al. 2006, MNRAS, 366, 1151

Sarzi, M., Shields, J. C., Schawinski, K., et al. 2010, MNRAS, 402, 2187

Schoenmakers, R. H. M., Franx, M., \& de Zeeuw, P. T. 1997, MNRAS, 292, 349

Shlosman, I., Frank, J., \& Begelman, M. C. 1989, Nature, 338, 45

Tully, R. B., Rizzi, L., Shaya, E. J., et al. 2009, AJ, 138, 323

van der Hulst, J. M., Terlouw, J. P., Begeman, K. G., Zwitser, W., \& Roelfsema, P. R. 1992, in Astronomical Data Analysis Software and Systems I, eds. D. M. Worrall, C. Biemesderfer, \& J. Barnes, ASP Conf. Ser., 25, 131

Van der Laan, T. P. R., Schinnerer, E., Boone, F., et al. 2011, A\&A, 529, A45

Van der Laan, T. P. R., Schinnerer, E., Emsellem, E., et al. 2013, A\&A, 551, A81

Vázquez, G. A., \& Leitherer, C. 2005, ApJ, 621, 695

Vogelaar, M. G. R., \& Terlouw, J. P. 2001, in Astronomical Data Analysis Software and Systems X, eds. F. R. Harnden, Jr., F. A. Primini, \& H. E. Payne, ASP Conf. Ser., 238, 358

Yuan, C., \& Yang, C.-C. 2006, ApJ, 644, 180 